\title{
General Dynamics of Pulsed Digital Oscillators
}

\author{
Manuel Domínguez, Member, IEEE, Joan Pons-Nin, Member, IEEE, and Jordi Ricart
}

\begin{abstract}
The objective of this work is to analyze pulsed digital oscillators (PDOs), as dynamical systems. It is proved that under some conditions, the bitstream at the output of the oscillator is that of the sign of a sampled sinusoid at the resonant frequency of the resonator, and that a bijection exists between these sequences (without distinguishing between a sequence and its negated version) and those of first-order sigma-delta modulators. This provides a new and simple method of obtaining the oscillation frequency of PDOs just from their bitstream.
\end{abstract}

Index Terms-Oscillators, pulsed digital oscillators (PDOs), sigma-delta $(\Sigma \Delta)$ modulation.

\section{LIST OF SYMBOLS}

$\begin{array}{ll}z^{*} & \text { Complex conjugate, } z \in \mathbb{C} . \\ \langle a\rangle & a \text { modulus } 1, a \in \mathbb{R} . \\ \lfloor a\rfloor & \text { Largest integer less or equal to } a, a \in \mathbb{R} . \\ \lceil a\rceil & \text { Smallest integer not less than } a, a \in \mathbb{R} . \\ \operatorname{sign}(a) & \text { Sign function, } a \in \mathbb{R} . \\ \operatorname{Real}(b) & \text { Real part of } b \in \mathbb{C} . \\ \operatorname{sgn}(b) & \text { sign }(\operatorname{Real}(b)), b \in \mathbb{C} . \\ A \backslash B & \{x \in A: x \notin B\} . \\ \text { g.c.d. }(M, N) & \text { Greater common divider, } M, N \in \mathbb{N} .\end{array}$

\section{INTRODUCTION}

$\mathbf{T}$ HE objective of this work is to analyze the dynamics of pulsed digital oscillators (PDOs) [1]-[4], seen as dynamical systems. Microelectromechanical systems are well known for presenting nonlinearities in actuation and/or sensing, [5], [6]. PDOs are simple circuits that can easily overcome some of these nonlinearities. The PDOs general circuit topology can be seen in Fig. 1. It is a sampled circuit and at each sampling time it is only necessary to know if the resonator is above or below its rest position. The actuation force fed into the resonator consists of a train of deltas taking values $\{+F,-F\}$ or $\{+F, 0\}$. In practical realizations a train of short pulses is used instead. The output of the system is the bitstream provided by the 1-bit quantizer (the sign of the sampled position of the resonator). Although

Manuscript received February 16, 2007; revised September 15, 2007. First published February 8, 2008; last published August 13, 2008 (projected). This work was supported in part by DPI2004-06756-C03-02 and TEC-67951/MIC. This paper was recommended by Associate Editor C. W. Wu.

The authors are with MNT Micro and Nano Technologies Group, 08034 Barcelona, Spain (e-mail: mpumar@eel.upc.edu).

Digital Object Identifier 10.1109/TCSI.2008.918175

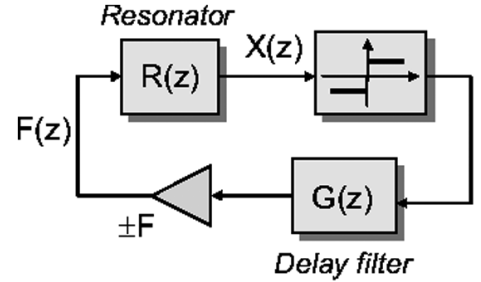

Fig. 1. General circuit topology of PDOs.

the influence of the filter $G(z)$ has been analyzed in [4] to some extent, in this work we will assume for the sake of simplicity that $G(z)=z^{-L}, L \in \mathbb{N}$.

These circuits can be seen as iterative piecewise-linear maps, basically in the complex plane, although other memory structures will be added to account for the delay blocks in the feedback loop. Previous works have been focused on the symbolic dynamics of piecewise-linear maps [7]-[11], but the specifity of the results we are looking for, requires an extensive and particular analysis of the dynamics of PDOs.

The main question that this work tries to solve is: what kind of sequences are at the output of PDOs? and what phase-space trajectories does the resonator follow? These are very similar questions to those solved in [12], [13] for first-order sigma-delta $(\Sigma \Delta)$ modulators. The results obtained in this work are the first rigorous results proving that under some conditions PDOs are actually oscillators, explaining the phase-space trajectories of the resonator and providing the bitstreams at the output. It will be shown that PDOs are much more related to first-order modulators than to bandpass $\Sigma \Delta$ modulators, as had been pointed out in [2], and that the typical quantization noise shaping of standard $\Sigma \Delta$ modulators must not be expected at the output of PDOs.

We will assume that the resonator can be described by the following equation:

$$
m \frac{d^{2} x(t)}{d t^{2}}+b \frac{d x(t)}{d t}+k x(t)=F(t)
$$

where $m$ is the mass of the resonator, $b$ is damping factor, $k$ is the spring factor and $F(t)$ is the external force being applied on the resonator. Now, what kind of sequences are at the output of PDOs? Let us for a moment look to Fig. 1, and assume that the oscillator is working 'well'. It would mean that the resonator position waveform would be a sinusoid, and therefore the bitstream at the output of the PDO would be the sign of a sampled sinusoid, from here on $S S S=S^{3}$ sequences

$$
b_{n}=\operatorname{sign}\left(\cos \left(2 \pi f n+\phi_{0}\right)\right), \quad \phi_{0} \in[0,2 \pi)
$$

for a given normalized frequency, $f$. On the other hand, one of the usual similes used to describe the behaviour of first-order 
$\Sigma \Delta$ modulators is the rotations of the circle, a very similar situation to the one described above, taking into account both variables of the resonator: position and velocity. The main difference is that in the $\Sigma \Delta$ case, a ' 1 ' is emitted each sampling time a complete rotation of the circle has been accomplished, whereas in the PDO case, at each sampling time a ' 1 ' or ' -1 ' is emitted depending on whether the resonator is in a given half of the circle or in the other.

The main results of this paper, stated in the form of 3 theorems, can be summarized as follows:

a) There is a bijection between $S^{3}$ sequences (without distinguishing between a sequence and its negated version) and first-order $\Sigma \Delta$ sequences. This will provide a new simple way of obtaining the oscillation frequency of the resonator, directly from the bitstream at the output of the PDO.

b) Under some sufficient conditions, PDOs will have $S^{3}$ sequences at their output, at the resonant frequency of the resonator.

Section II will provide the relationship between first-order $\Sigma \Delta$ sequences and $S^{3}$ sequences. Section III will present the PDO as an iterative map. Section IV will provide the tools to graphically observe under which conditions we have perfect $S^{3}$ sequences at the output of the PDO, and the relation with the initial condition of the resonator. Section V, based on the intuition gained by the extensive analysis of the results of Section IV, will provide general properties of PDO systems and conditions under which PDOs produce $S^{3}$ sequences. Finally, the bitstream spectra of $S^{3}$ sequences are analyzed in the Appendix.

\section{First ORder $\Sigma \Delta$ Modulators AND $S^{3}$ Bitstreams}

The objective of this section is to establish the relation between the Sign of a Sampled Sinusoid sequences and first-order $\Sigma \Delta$ sequences. $S^{3}$ sequences can be described as

$$
s(n)=\operatorname{sign}(\sin (2 \pi f n+2 \pi \lambda)), \quad n \geq 0
$$

with $\lambda \in[0,1)$, and being $f \in[0,1 / 2)$ a given normalized frequency. Any $S^{3}$ sequence generated with a frequency $f_{0} \in$ $[1 / 2,1)$, and offset $\lambda_{0} \in[0,1)$ is identical to that generated with a frequency $1-f_{0}$ and offset $\left\langle 1 / 2-\lambda_{0}\right\rangle$.

These sequences can also be expressed in the following way:

$$
\frac{s(n)}{2}=\langle n f-1 / 2+\lambda\rangle-\langle n f+\lambda\rangle, \quad n \geq 0 .
$$

On the other hand, first-order $\Sigma \Delta$ modulators produce sequences of the form [13]

$$
b(n)=x+\langle(n-1) x+\lambda\rangle-\langle n x+\lambda\rangle, \quad n \geq 0
$$

for some given parameters $x, \lambda \in[0,1)$. The set of all $\Sigma \Delta$ sequences will be named $\mathcal{S D}$. The set of all unordered pairs $\{s,-s\}$ of $S^{3}$ sequences, will be named $\mathcal{S}^{3}$.

Theorem 1: A bijection exists between the set $\mathcal{S}^{3}$ and the set of first-order $\Sigma \Delta$ sequences, $\mathcal{S D}$.
Proof: Given a pair $\{s,-s\} \in \mathcal{S}^{3}$, let us assume without loss of generality that $s$ can be expressed in the form

$$
s(n)=2(\langle n f-1 / 2+\lambda\rangle-\langle n f+\lambda\rangle), \quad n \geq 0
$$

with $f \in[0,1 / 2)$ and $\lambda \in[0,1 / 2)$ (if $\lambda$ needs to be in $[1 / 2,1$ ), then $-s$ will have $\lambda$ in the desired segment). Let us now define a function $R: \mathcal{S}^{3} \rightarrow \mathcal{S D}$, such that for any $\{s,-s\} \in$ $\mathcal{S}^{3}, R(\{s,-s\})=b$ where sequence $b$ is defined as $b(n)=$ $(1 / 2)|s(n)-s(n-1)|, n \geq 0$ and where $s(-1)$ is defined as $s(-1)=1 / 2(\langle-f-1 / 2+\lambda\rangle-\langle-f+\lambda\rangle)$.

If the sequence $s$ is aperiodic then $f \in \mathbb{R}-\mathbb{Q}$ and therefore the choice of parameters $f, \lambda$ is unique. If the sequence is periodic, then parameter $f=M / N \in \mathbb{Q}$, with $g . c . d .(M, N)=1$, is unique but $\lambda$ can take values over a segment of length $1 / N$ for $N$ even, and $1 / 2 N$ for $N$ odd [3]. This collection of segments is, for $\mathrm{N}$ even, $\{[i / N,(i+1) / N): i=0, \cdots, N / 2-1\}$ and, for $\mathrm{N}$ odd, $\{[i / 2 N,(i+1) / 2 N): i=0, \cdots, N-1\}$.

From (6), $s(n)=1$ when $\langle n f+\lambda\rangle \in[0,1 / 2)$ and $s(n)=-1$ if $\langle n f+\lambda\rangle \in[1 / 2,1)$.

Now, if $s(n)=-s(n-1)$, then

$$
f+((n-1) f+\lambda) \bmod \frac{1}{2}-(n f+\lambda) \bmod \frac{1}{2}=\frac{1}{2}
$$

which is the same as

$$
2 f+\langle(n-1) 2 f+2 \lambda\rangle-\langle n 2 f+2 \lambda\rangle=1 .
$$

On the other hand, if $s(n)=s(n-1)$, then

$$
f+((n-1) f+\lambda) \bmod \frac{1}{2}-(n f+\lambda) \bmod \frac{1}{2}=0
$$

which means that

$$
2 f+\langle(n-1) 2 f+2 \lambda\rangle-\langle n 2 f+2 \lambda\rangle=0 .
$$

Therefore, the sequence generated through the detection of edges in $s$ belongs to $\mathcal{S D}$, i.e., $b(n)=(1 / 2)|s(n)-s(n-1)| \in$ $\mathcal{S D}$. And $b(n)$ is an $\mathcal{S D}$ sequence generated by parameters $2 f$ and $2 \lambda$. This result does not depend on the choice of $\lambda$ when $f$ is rational, because for $N$ even, the $N / 2$ segments, namely $\{[i / N,(i+1) / N): i=0, \cdots, N / 2-1\}$ are transformed into $\{[i / N / 2,(i+1) / N / 2): i=0, \cdots, N / 2-1\}$, which are exactly the uncertainty offset segments for sequence $b$, because its main parameter is $2 f=M /(N / 2)(N$ is even, and now g.c.d. $(M, N / 2)=1)$. A very similar situation occurs for $N$ odd: the uncertainty segments of signal $s,\{[i / 2 N,(i+1) / 2 N)$ : $i=0, \cdots, N-1\}$, become $\{[i / N,(i+1) / N): i=0, \cdots, N-$ $1\}$, which are exactly the segments corresponding to sequence $b$, of parameter $2 f=2 M / N$. Therefore, function $R$ is injective.

Now, given a sequence $b \in \mathcal{S D}$, it can be expressed in the form

$$
b(n)=x+\langle(n-1) x+\lambda\rangle-\langle n x+\lambda\rangle
$$

with $x, \lambda \in[0,1)$. We generate the sequence $s$ as follows: 


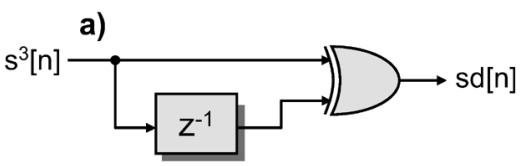

b)

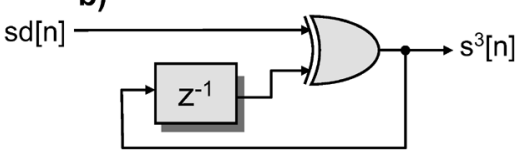

Fig. 2. Circuits for conversion between $S^{3}$ and $S D$ bitstreams. a) from $S^{3}$ to $S D$ sequences, b) from $S D$ to $S^{3}$ sequences. A change on the initial condition of the delay block of circuit b) generates a change from an $s \in S^{3}$ sequence to $-s$.

a) $s(0)= \begin{cases}1, & \text { if } b(0)=0 \\ -1, & \text { if } b(0)=1\end{cases}$

b) for $n>0, s(n)= \begin{cases}s(n-1), & \text { if } b(n)=0 \\ -s(n-1), & \text { if } b(n)=1 .\end{cases}$

Now, if $b(n)=0$ it means that

$\frac{x}{2}+\left((n-1) \frac{x}{2}+\frac{\lambda}{2}\right) \bmod \frac{1}{2}-\left(n \frac{x}{2}+\frac{\lambda}{2}\right) \bmod \frac{1}{2}=0$

and therefore if $\langle n(x / 2)+(\lambda / 2)\rangle \in[0,1 / 2)$ then $\langle(n-$ $1)(x / 2)+(\lambda / 2)\rangle \in[0,1 / 2) ;$ and if $\langle n(x / 2)+(\lambda / 2)\rangle \in[1 / 2,1)$ then $\langle(n-1)(x / 2)+(\lambda / 2)\rangle \in[1 / 2,1)$. Following the same reasoning, if $b(n)=1$ then if $\langle n(x / 2)+(\lambda / 2)\rangle \in[0,1 / 2)$ then $\langle(n-1)(x / 2)+(\lambda / 2)\rangle \in[1 / 2,1)$; and if $\langle n(x / 2)+(\lambda / 2)\rangle \in$ $[1 / 2,1)$ then $\langle(n-1)(x / 2)+(\lambda / 2)\rangle \in[0,1 / 2)$.

This in fact implies that the bits in $s$ only change their value when $\langle n(x / 2)+(\lambda / 2)\rangle$ changes from taking values in $[0,1 / 2)$ to $[1 / 2,1)$ or vice versa. Therefore, either

$$
s(n)= \begin{cases}1, & \text { if }\left\langle n \frac{x}{2}+\frac{\lambda}{2}\right\rangle \in[0,1 / 2) \\ -1, & \text { if }\left\langle n \frac{x}{2}+\frac{\lambda}{2}\right\rangle \in[1 / 2,1)\end{cases}
$$

or

$$
s(n)= \begin{cases}-1, & \text { if }\left\langle n \frac{x}{2}+\frac{\lambda}{2}\right\rangle \in[0,1 / 2) \\ 1, & \text { if }\left\langle n \frac{x}{2}+\frac{\lambda}{2}\right\rangle \in[1 / 2,1)\end{cases}
$$

which means that $\{s,-s\} \in \mathcal{S}^{3}$. Following the same reasoning as before, the above result does not depend on the choice of parameter $\lambda$ for $x$ rational, and $R(\{s,-s\})=b$. Therefore, we may finally conclude that function $R$ is also surjective, and therefore it is a bijection.

The above result provides a very simple and practical way of transforming $S^{3}$ sequences into $S D$ and vice versa (see Fig. 2). Simulation and experimental results confirm that the circuit of Fig. 2(a) can be used as an intermediate step to extract the oscillation frequency directly from general PDO bitstreams.

\section{PDOS AS ITERATIVE MAPS}

The objective of this section is to see the discrete time dynamics of PDOs as iterative maps. The general circuit topology of the PDO can be seen in Fig. 1. The most common form of (1) is

$$
x^{\prime \prime}(t)+2 \rho \omega_{0} x^{\prime}(t)+\omega_{0}^{2} x(t)=\frac{F(t)}{m}
$$

where

$$
\rho=\frac{b}{2 \sqrt{k m}} \quad \frac{\omega_{0}}{2 \pi}=f_{0}=\frac{1}{2 \pi} \sqrt{\frac{k}{m}} .
$$

The evolution of the Pulsed Digital Oscillator can be seen as an example of systems governed by Impulsive Differential Equations [14]. This means, that between any two sampling events, the resonator moves freely along its rotating trajectory and at each sampling event, its velocity is instantaneously changed by the force delta being applied on it. In this sense, if we define the sequence $\left\{x_{n}, v_{n}\right\}=\left\{x\left(n T_{s}\right), x^{\prime}\left(n T_{s}\right)\right\}$, where $T_{s}=1 / f_{s}$ is the period of the sampling clock, then the evolution of the oscillator for $n \geq 0$ is governed by the following equations:

$$
\left(\begin{array}{l}
x_{n+1} \\
v_{n+1}
\end{array}\right)=\alpha\left(\begin{array}{ll}
a_{11} & a_{12} \\
a_{21} & a_{22}
\end{array}\right)\left(\begin{array}{l}
x_{n} \\
v_{n}
\end{array}\right) \mp \frac{F}{m}\left(\begin{array}{c}
0 \\
q_{n-L}
\end{array}\right)
$$

where

$$
\begin{aligned}
\alpha & =e^{-2 \pi \frac{\rho}{\sqrt{1-\rho^{2}}} f} \\
a_{11} & =\cos (2 \pi f)+\frac{\rho}{\sqrt{1-\rho^{2}}} \sin (2 \pi f) \\
a_{12} & =\frac{1}{\omega_{0} \sqrt{1-\rho^{2}}} \sin (2 \pi f) \\
a_{21} & =-\frac{\omega_{0}}{\sqrt{1-\rho^{2}}} \sin (2 \pi f) \\
a_{22} & =\cos (2 \pi f)-\frac{\rho}{\sqrt{1-\rho^{2}}} \sin (2 \pi f)
\end{aligned}
$$

and $f=\left(f_{0} / f_{S}\right) \sqrt{1-\rho^{2}}, q_{n}=\operatorname{sign}\left(x_{n}\right)$. The initial conditions are $x_{0}, v_{0}$ and $\left\{b_{i}:-L \leq i \leq-1\right\}$.

In order to handle more comfortable expressions it is convenient to work on the complex plane with an appropriate normalization. This will allow us later to use Fourier Transform notation to find necessary conditions to obtain $S^{3}$ sequences in PDOs. In this way, by defining the following variable sequence:

$$
u_{n}=x_{n}+\frac{j}{\sqrt{1-\rho^{2}}}\left(\rho x_{n}+\frac{v_{n}}{\omega_{0}}\right)
$$

the evolution of the system is described through this equation

$$
u_{n+1}=p u_{n}-j \delta q_{n-L}, \quad u_{n} \in \mathbb{C}
$$

where $\delta= \pm\left(F / m \omega_{0} \sqrt{1-\rho^{2}}\right.$, depending on the sign of the feedback loop of the oscillator, and $p=\alpha e^{-j 2 \pi f}$, being $q_{n}=$ $\operatorname{sign}\left(\operatorname{Real}\left(u_{n}\right)\right)$.

In order to consider this system as a map $(\tau)$, we must define our phase space. Let $\mathcal{B}=\{+1,0,-1\} \subset \mathbb{R}$. The most straightforward option for $L>0$ is

$$
\begin{array}{rcc}
\mathbb{C} \times \mathcal{B}^{L} & \rightarrow & \mathbb{C} \times \mathcal{B}^{L} \\
\left(u, b_{1}, \cdots, b_{L}\right) & & \left(p u-j \delta b_{L}, \operatorname{sgn}(u), b_{1}, \cdots, b_{L-1}\right)
\end{array}
$$

where $u \in \mathbb{C}, b_{i} \in \mathcal{B}, 1 \leq i \leq L$. For $L=0$

$$
\begin{aligned}
& \tau: \mathbb{C} \rightarrow \mathbb{C} \\
& u \quad p u-j \delta \operatorname{sgn}(u)
\end{aligned}
$$

where $\operatorname{sgn}(u), u \in \mathbb{C}$ is defined as $\operatorname{sign}(\operatorname{Real}(u))$. 
We will consider that $\tau^{0}(z), z \in \mathbb{C} \times \mathcal{B}^{L}$, is the identity and that $\tau^{n}(z)$ is the result of applying $n$ times function $\tau$ to $z$, $n>0$.

Definition 1: The projections $\pi_{0}: \mathbb{C} \times \mathcal{B}^{L} \rightarrow \mathbb{C}$ and $\pi_{i}: \mathbb{C} \times$ $\mathcal{B}^{L} \rightarrow \mathcal{B}, 1 \leq i \leq L$ are defined as $\pi_{0}\left(\left(u, b_{1}, \cdots, b_{L}\right)\right)=u$, and $\pi_{i}\left(\left(u, b_{1}, \cdots, b_{L}\right)\right)=b_{i}$.

In order to simplify notation we further define as follows.

Definition 2: The function $T_{n}(z): \mathbb{C} \times \mathcal{B}^{L} \rightarrow \mathbb{C}, n \geq 0$ is defined as $\pi_{0}\left(\tau^{n}(z)\right)$.

Definition 3: In the case $L \geq 1$, the functions $b_{n}^{i}(z): \mathbb{C} \times$ $\mathcal{B}^{L} \rightarrow \mathcal{B}$ are defined as $\pi_{i}\left(\tau^{n}(z)\right), 1 \leq i \leq L, n \geq 0$. If $L=0$, $b_{n}^{0}(z)$ is defined as $\operatorname{sgn}\left(T_{n}(z)\right), n \geq 0$.

If $L \geq 1$ the feedback loop of the oscillator ensures that $b_{n}^{i}(z)=\operatorname{sgn}\left(T_{n-i}(z)\right), 1 \leq i \leq L, n \geq 0, n-i \geq 0$. If $n-i<0$ then $b_{n}^{i}(z)=\pi_{|n-i|}(z)$.

\section{Symbolic DYNAMICS OF THE PDO}

The purpose of this section is to study how the initial conditions of the resonator and the $L$ initial bits affect the future evolution of the oscillator, specified by $\delta, f, \rho, L$. Through the values taken by $f$ and $\rho$ (or $\alpha$ ), $p$ is specified.

The result of this section is a partition on the complex plane, $\mathcal{P}(\mathbf{b})$, depending on the initial conditions of the bitstream $\mathbf{b}=$ $\left(b_{1}, \cdots, b_{L}\right), \mathbf{b} \in \mathcal{B}^{L}$, and a function, $G: \mathcal{P}(\mathbf{b}) \rightarrow \mathbb{N} \cup\{\infty\}$, such that for any $u \in P \in \mathcal{P}(\mathbf{b})$ there is a $u^{\prime} \in P$ such that $\operatorname{sgn}\left(T_{i}((u, \mathbf{b}))\right)=\operatorname{sgn}\left(T_{i}\left(\left(u^{\prime}, \mathbf{b}\right)\right)\right)=\operatorname{sgn}\left(p^{i} u^{\prime}\right)$ for $0 \leq i \leq$ $G(P, \mathbf{b})$, being $G(P, \mathbf{b})$ the maximum number of clock cycles on which it is possible to find $u^{\prime} \in \mathbb{C}$ with this property. The idea of generating a partition consistent with the bitstream has been used in the past, for example in [15].

Definition 4: The function $H_{n}((u, \mathbf{b})): \mathbb{C} \times \mathcal{B}^{L} \rightarrow \mathbb{C}$ is defined as $H_{n}((u, \mathbf{b}))=T_{n}((u, \mathbf{b}))-p^{n} u, n>0$, with $H_{0}(z)=0$.

The time evolution of the resonator can be described with the following equation:

$$
T_{n}(z)=p^{n} u+H_{n}(z), z=(u, \mathbf{b}) \in \mathbb{C} \times \mathcal{B}^{L}, n \geq 0
$$

and

$$
H_{n}(z)=-j \delta p^{n-1} \sum_{i=0}^{n-1} p^{-i} b_{i}^{L}(z)
$$

The feedback loop of the oscillator ensures that $b_{i}^{1}(z)=$ $\operatorname{sgn}\left(T_{i-1}(z)\right), b_{i+1}^{k}(z)=b_{i}^{k-1}, 2 \leq k \leq L, i>0$.

For a given initial condition of the bitstream, $\mathbf{b}=$ $\left(b_{1}, \cdots, b_{L}\right)$, Lemmas 1,2 and Corollary 1 will provide a sequence of partitions of the complex plane $\mathcal{P}_{n}(\mathbf{b})$ such that for each one of the sets of these partitions, the initial conditions of the resonator are undistinguishable from the first $n$ bits at the output of the oscillator.

Lemma 1: If $z=(u, \mathbf{b}), z^{\prime}=\left(u^{\prime}, \mathbf{b}\right) \in \mathbb{C} \times \mathcal{B}^{L}$, it is $\operatorname{sgn}\left(T_{i}(z)\right)=\operatorname{sgn}\left(T_{i}\left(z^{\prime}\right)\right)$ for $0 \leq i \leq n$ if and only if $u=$ $j p^{-i} \lambda_{i}-p^{-i} H_{i}(z)+r_{i} p^{-i}$ and $u^{\prime}=j p^{-i} \lambda_{i}^{\prime}-p^{-i} H_{i}(z)+r_{i}^{\prime} p^{-i}$ for some $\lambda_{i}, \lambda_{i}^{\prime}, r_{i}, r_{i}^{\prime} \in \mathbb{R}$ with $\operatorname{sign}\left(r_{i}\right)=\operatorname{sign}\left(r_{i}^{\prime}\right), 0 \leq i \leq n$.

Proof: The numbers $\lambda_{i}, r_{i}$ are uniquely determined as the imaginary and real parts respectively of the complex numbers $p^{i} u+H_{i}(z), 0 \leq i \leq n$. Parameters $\lambda_{i}^{\prime}, r_{i}^{\prime}$ are the imaginary and real parts of $p^{i} u^{\prime}+H_{i}(z), 0 \leq i \leq n$, note that they are defined as a function of $H_{i}(z)$, not of $H_{i}\left(z^{\prime}\right)$. Now, it is $H_{i}(z)=H_{i}\left(z^{\prime}\right)$, $0 \leq i \leq L$, because they all depend only on $\mathbf{b} \in \mathcal{B}^{L},\left(\pi_{i}(z)=\right.$ $\left.\pi_{i}\left(z^{\prime}\right), i=1 \cdots, L\right)$. Therefore, $\operatorname{sgn}\left(T_{i}(z)\right)=\operatorname{sgn}\left(T_{i}\left(z^{\prime}\right)\right)$, $0 \leq i \leq \operatorname{Min}(n, L)$, if and only if $\operatorname{sign}\left(r_{i}\right)=\operatorname{sgn}\left(p^{i} u+\right.$ $\left.H_{i}(z)\right)=\operatorname{sign}\left(r_{i}^{\prime}\right)=\operatorname{sgn}\left(p^{i} u^{\prime}+H_{i}(z)\right), 0 \leq i \leq \operatorname{Min}(n, L)$. If $n \leq L$ we are done.

Let us assume that $n>L$. Now $H_{L+1}(z)=H_{L+1}\left(z^{\prime}\right)$ because $\operatorname{sgn}(u)=b_{L}^{L}(z)=\operatorname{sgn}\left(u^{\prime}\right)=b_{L}^{L}\left(z^{\prime}\right)$. Taking this into account, it is $\operatorname{sgn}\left(T_{L+1}(z)\right)=\operatorname{sgn}\left(T_{L+1}\left(z^{\prime}\right)\right)$ if and only if $\operatorname{sign}\left(r_{L+1}\right)=\operatorname{sgn}\left(p^{L+1} u+H_{L+1}(z)\right)=$ $\operatorname{sign}\left(r_{L+1}^{\prime}\right)=\operatorname{sgn}\left(p^{L+1} u^{\prime}+H_{L+1}(z)\right)$. By proceeding recursively we will reach $\operatorname{sgn}\left(T_{i}(z)\right)=\operatorname{sgn}\left(T_{i}\left(z^{\prime}\right)\right)$ and $H_{i+L}(z)=H_{i+L}\left(z^{\prime}\right)$, for $i=0, \cdots, n$.

This result can be interpreted as follows: given an initial $\mathbf{b} \in$ $\mathcal{B}^{L}$ and an initial $u \in \mathbb{C}$, it is possible to obtain the future evolution of the bitstream at the output of the oscillator. This sequence of bits determines the values of $H_{i}((u, \mathbf{b}))$, which also determine the lines $l_{i}((u, \mathbf{b}))=\left\{\xi \in \mathbb{C}: \xi=j p^{-i} \lambda-\right.$ $\left.p^{-i} H_{i}(z), \lambda \in \mathbb{R}\right\}$. Then, Lemma 1 states that for those initial conditions $\left(u^{\prime}\right)$ located at the same sides of the lines $l_{i}((u, \mathbf{b}))$, $0 \leq i \leq n$ as $u$, the output of the oscillator is the same as in the case of $u$, for at least $n$ clock cycles.

The next step is to generate a sequence of partitions $\left(\mathcal{P}_{n}(\mathbf{b})\right)$, such that for any $u, u^{\prime} \in P \in \mathcal{P}^{n}(\mathbf{b})$, it is $\operatorname{sgn}\left(T_{i}((u, \mathbf{b}))\right)=$ $\operatorname{sgn}\left(T_{i}\left(\left(u^{\prime}, \mathbf{b}\right)\right)\right), 0 \leq i \leq n .:$

Lemma 2: For a given $n \geq 0$ and $\mathbf{b} \in \mathcal{B}^{L}$, it is possible to construct a set of partitions $\mathcal{P}^{k}(\mathbf{b}), 0 \leq k \leq n$, in $\mathbb{C}$ such that for all $u, u^{\prime} \in P \in \mathcal{P}^{k}(\mathbf{b})$, it is $\operatorname{sgn}\left(T_{i}((u, \mathbf{b}))\right)=$ $\operatorname{sgn}\left(T_{i}\left(\left(u^{\prime}, \mathbf{b}\right)\right)\right), i=0, \cdots, k$.

Proof: We will proceed to construct this partition through an algorithm.

\section{Step 1:}

The line $\left.\left\{l_{0}((u, \mathbf{b}))=j \lambda\right) \subset \mathbb{C}, \lambda \in \mathbb{R}\right\}$ will be used to generate three different connected sets: $A=\{u \in$ $\mathbb{C}: \operatorname{Real}(u)<0\}, B=\{u \in \mathbb{C}: \operatorname{Real}(u)>0\}$, $C=\{u \in \mathbb{C}: \operatorname{Real}(u)=0\}$. The sets $A, B, C$ will be the ones in $\mathcal{P}^{0}(\mathbf{b})$. We define the counter variable $\mathrm{K}=0$. Step 2:

For each set $P \in \mathcal{P}^{K}(\mathbf{b})$ we choose a point $v \in P$ and calculate $H_{K+1}((v, \mathbf{b}))$. Of course the choice of $v \in P$ is of no relevance.

If $l_{K+1}(v, \mathbf{b}) \cap P=\emptyset$ then $P \in \mathcal{P}^{K+1}$. If not, the connected components of $P \backslash l_{K+1}(v, \mathbf{b})$ will be included in $\mathcal{P}^{K+1}(\mathbf{b})$, together with $P \cap l_{K+1}(v, \mathbf{b})$.

Next, we proceed with the following set in $\mathcal{P}^{K}(\mathbf{b})$. When all the sets in the partition $\mathcal{P}^{K}(\mathbf{b})$ have undergone this process, if $K=n-1$ the algorithm ends and if not we repeat Step 2, while increasing the value of $K$.

\section{Corollary 1: $\mathcal{P}^{n+1}$ is a partition finer than $\mathcal{P}^{n}$.}

Proof: Each set of $\mathcal{P}^{n}(\mathbf{b})$ is either obtained as the result of a subdivision of a set in $\mathcal{P}^{n-1}(\mathbf{b})$ or it belongs to $\mathcal{P}^{n-1}(\mathbf{b})$.

Now we will construct a new partition, $\mathcal{P}(\mathbf{b})$, based on the sequence $\mathcal{P}_{n}(\mathbf{b})$. 
Definition 5: Given an oscillator and a $z=(u, \mathbf{b}) \in \mathbb{C} \times \mathcal{B}^{L}$, the collection of sets $F Z^{i}(z) \subset \mathbb{C}, i \in \mathbb{N}$, is defined as

$$
\begin{aligned}
F Z^{i}(z)=\left\{u \in \mathbb{C}: u=j p^{-i} \lambda-H_{i}(z) p^{-i} r_{0},\right. & \\
\lambda & \left.\quad \in \mathbb{R}, r_{0} \in[0,1]\right\}
\end{aligned}
$$

and if $\operatorname{Real}\left(H_{i}(z)\right)=0$ then $F Z^{i}(z)=\emptyset$.

Lemma 3: Given a $z=(u, \mathbf{b}) \in \mathbb{C} \times \mathcal{B}^{L}$, and $i \geq 0$, it is $\left.\operatorname{sgn}\left(T_{i}(z)\right)=-\operatorname{sgn}\left(p^{i} u\right)\right)$ if and only if $u \in F Z^{i}(z)$.

Proof: If $\operatorname{Real}\left(H_{i}(z)\right)=0$ then it is always $\operatorname{sgn}\left(T_{i}(z)\right)=$ $\left.\operatorname{sgn}\left(p^{i} u\right)\right)$. On the other hand, if $\operatorname{Real}\left(H_{i}(z)\right) \neq 0$, it is possible to find $\lambda_{0}, r_{0} \in \mathbb{R}$, such that $u=j p^{-i} \lambda_{0}-p^{-i} H_{i}(z) r_{0}$. Then $\operatorname{sgn}\left(T_{i}(z)\right)=\operatorname{sign}\left(\operatorname{Real}\left(H_{i}(z)\right)\left(1-r_{0}\right)\right) \neq$ $\operatorname{sgn}\left(p^{i} u\right)=-\operatorname{sign}\left(\operatorname{Real}\left(H_{i}(z)\right) r_{0}\right)$, if only if, $r_{0} \in[0,1]$.

Corollary 7: Given a $z=(u, \mathbf{b}) \in \mathbb{C} \times \mathcal{B}^{L}$, it is $\left.\operatorname{sgn}\left(T_{i}(z)\right)=\operatorname{sgn}\left(p^{i} u\right)\right), 0 \leq i \leq n$ if and only if $u \notin \bigcup_{i=0}^{n} F Z^{i}(z), 0 \leq i \leq n$.

Obviously, given a $\mathbf{b} \in \mathcal{B}^{L}$, for any two $u, u^{\prime} \in P \in \mathcal{P}^{n}(\mathbf{b})$, $n \geq 0$, it is $F Z^{i}((u, \mathbf{b}))=F Z^{i}\left(\left(u^{\prime}, \mathbf{b}\right)\right), 0 \leq i \leq n+1$.

Lemma 4: For any given $n \geq 0$ and $z=(u, \mathbf{b}) \in \mathbb{C} \times \mathcal{B}^{L}$, it is $\operatorname{sgn}\left(T_{i}(z)\right)=\operatorname{sgn}\left(p^{i} u\right), 0 \leq i \leq n$ if and only if $u \in$ $P \in \mathcal{P}^{n}(\mathbf{b})$, and $u \notin \cup_{i=0}^{n} F Z^{i}(u, \mathbf{b})$. If $u \in \cup_{i=0}^{n} F Z^{i}(u, \mathbf{b})$ but there is a $u^{\prime} \in P$ such that $u^{\prime} \notin \cup_{i=0}^{n} F Z^{i}(u, \mathbf{b})$ then $\operatorname{sgn}\left(T_{i}(z)\right)=\operatorname{sgn}\left(p^{i} u^{\prime}\right), 0 \leq i \leq n$.

Proof: It is evident from Corollary 2 and Lemmas 1 and 2 and taking into account that if $u, u^{\prime} \in P \in \mathcal{P}^{n}(\mathbf{b}), n \geq 0$, it is $\cup_{i=0}^{n} F Z^{i}(u, \mathbf{b})=\cup_{i=0}^{n} F Z^{i}\left(u^{\prime}, \mathbf{b}\right)$.

Definition 6: Given $b \in \mathcal{B}^{L}$ the collection of sets $\mathcal{P}(\mathbf{b})$ of $\mathbb{C}$ and the application $G: \mathcal{P}(\mathbf{b}) \times \mathcal{B}^{L} \rightarrow \mathbb{N} \cup\{\infty\}$ are defined in the following way. A subset $P \subset \mathbb{C}$ belongs to $\mathcal{P}(\mathbf{b})$ if $P \in$ $\mathcal{P}^{n}(\mathbf{b})$ and $P \subset \cup_{i=0}^{n} F Z^{i}(v, \mathbf{b}), v \in P$, for a given $n \geq 0$ and for all $Q_{i} \in \mathcal{P}^{i}(\mathbf{b}), i<n$, with $P \subset Q_{i}$ it is $Q_{i} \not{Z}$ $\cup_{k=0}^{i} F Z^{k}\left(v_{i}, \mathbf{b}\right), v_{i} \in Q_{i}$. Then $G(P, \mathbf{b})=n-1$.

For any sequence of sets $\left\{Q_{n} \in \mathcal{P}^{n}(\mathbf{b}), n \geq 0\right\}$ such that $Q_{n+1} \subset Q_{n}, Q_{n} \nsubseteq \cup_{i=0}^{n} F Z^{i}\left(v_{n}, \mathbf{b}\right), v_{n} \in Q_{n}$, and $Q=$ $\cap_{n=0}^{\infty} Q_{n}, Q \neq \emptyset$, then $Q \in \mathcal{P}(\mathbf{b})$, and $G(Q, \mathbf{b})=\infty$.

Lemma 5: The collection of sets $\mathcal{P}(\mathbf{b})$ is a partition of $\mathbb{C}$, and for any $P \in \mathcal{P}(\mathbf{b})$ it is $G(P, \mathbf{b}) \geq 1$.

Proof: For each $u \in \mathbb{C}$ there is a unique collection of sets $\left\{P_{n} \in \mathcal{P}^{n}(\mathbf{b})\right\}$ with $u \in P_{n}$, because each $\mathcal{P}^{n}(\mathbf{b})$ is a partition of $\mathbb{C}$. For each $n, P_{n+1} \subset P_{n}$ and $u \in \cap_{i=0}^{\infty} P_{i}$. If for all $n \geq 0$, $P_{n} \nsubseteq \cup_{i=0}^{n} F Z^{i}\left(v_{n}, \mathbf{b}\right), v_{n} \in P_{n}$ then $Q=\cap_{i=0}^{\infty} P_{i} \in \mathcal{P}(\mathbf{b})$ and $u \in Q$. Otherwise, if $n \geq 0$ is the minimum integer such that $P_{n} \subset \cup_{i=0}^{n} F Z^{i}\left(v_{n}, \mathbf{b}\right)$, for any $v_{n} \in P_{n}$, then $u \in P_{n} \in$ $\mathcal{P}(\mathbf{b})$. Therefore, for all $u \in \mathbb{C}$ there is a set $P \in \mathcal{P}(\mathbf{b})$ such that $u \in P$.

In order to see that for two different sets, $P, P^{\prime} \in \mathcal{P}(\mathbf{b})$, it is $P \cap P^{\prime}=\emptyset$, let us assume that $P \cap P^{\prime} \neq \emptyset, P \neq P^{\prime}$ and $G(P, \mathbf{b})=n, G\left(P^{\prime}, \mathbf{b}\right)=n^{\prime}$. It is not possible that $n=n^{\prime}$ because then they would belong to the same partition $\mathcal{P}_{n}$, and therefore $P=P^{\prime}$. If $P \in \mathcal{P}^{n+1}(\mathbf{b})$ and $P^{\prime} \in \mathcal{P}^{n^{\prime}+1}(\mathbf{b})$, assuming $n<n^{\prime}$, then $P^{\prime} \subset P$. But then $P \subset \cup_{i=0}^{n+1} F Z^{i}(v, \mathbf{b})$, $v \in P^{\prime} \subset P$, and $P^{\prime} \nsubseteq \cup_{i=0}^{n^{\prime}} F Z^{i}\left(v^{\prime}, \mathbf{b}\right), v^{\prime} \in P^{\prime}$, which is not possible because $n^{\prime}>n$ and $P^{\prime} \subset P$.

Now, let us assume $P, P^{\prime} \in \mathcal{P}(\mathbf{b})$, with $P \cap P^{\prime} \neq \emptyset$ with $G(P, \mathbf{b})=n$ and $G\left(P^{\prime}, \mathbf{b}\right)=\infty$. Then $P \in \mathcal{P}^{n+1}(\mathbf{b}), P \subset$ $\cup_{i=0}^{n+1} F Z^{i}(u, \mathbf{b}), u \in P$ and $P^{\prime}=\bigcap_{i=0}^{\infty} Q_{i}^{\prime}$, with $Q_{i}^{\prime} \in \mathcal{P}^{i}(\mathbf{b})$,

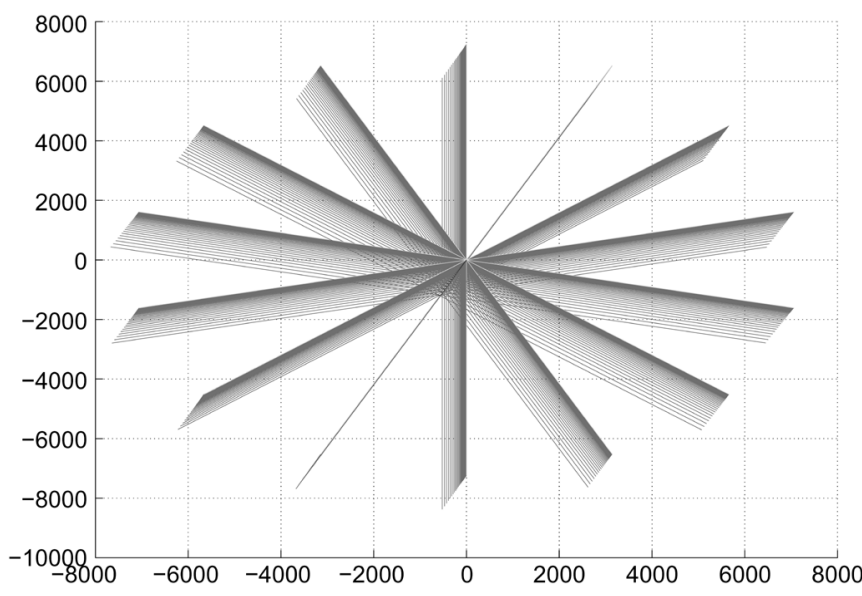

Fig. 3. Set of 250 lines $l_{0}(1) \cdots l_{249}(1)$, generated by $u=1$ for an oscillator with $f=3 / 7, L=0, \delta=1, \rho=0.005$. It is clear that point 1 is trapped by these lines and therefore if $1 \in P \in \mathcal{P}(\mathbf{b})$ then $G(P, \mathbf{b})<\infty$.

and $Q_{i} \nsubseteq \cup_{k=0}^{i} F Z^{k}\left(v_{i}, \mathbf{b}\right), v_{i} \in Q_{i}$. But it must be $P=$ $Q_{n+1}$, since $P \cap P^{\prime} \neq \emptyset$, and therefore we have a contradiction.

If $G(P, \mathbf{b})=G\left(P^{\prime}, \mathbf{b}\right)=\infty$, with $P \cap P^{\prime} \neq \emptyset$, then $P=$ $\cap_{i=0}^{\infty} Q_{i}$ and $P^{\prime}=\cap_{i=0}^{\infty} Q_{i}^{\prime}$, with $Q_{i}, Q_{i}^{\prime} \in \mathcal{P}^{i}(\mathbf{b})$. But it must be $Q_{i}=Q_{i}^{\prime}, i \geq 0$, since $P \cap P^{\prime} \neq \emptyset$. Therefore, $P=P^{\prime}$ and $\mathcal{P}(\mathbf{b})$ is a partition of $\mathbb{C}$.

Finally, $F Z^{0}(u, \mathbf{b})=F Z^{1}(u, \mathbf{b})=\emptyset$, because $\operatorname{Real}\left(H_{0}((u, \mathbf{b}))\right)=\operatorname{Real}\left(H_{1}((u, \mathbf{b}))\right)=0$, for any $u \in \mathbb{C}$. Therefore, $G(P, \mathbf{b}) \geq 1$ for any $P \in \mathcal{P}(\mathbf{b})$ and $\mathbf{b} \in \mathcal{B}^{L}$.

Lemma 6: Given $\mathbf{a} \mathbf{b} \in \mathcal{B}^{L}$, the following are true.

a) For each $u \in P \in \mathcal{P}(\mathbf{b})$, there is a $u^{\prime} \in P$ such that $\operatorname{sgn}\left(T_{i}(u, \mathbf{b})\right)=\operatorname{sgn}\left(T_{i}\left(u^{\prime}, \mathbf{b}\right)\right)=\operatorname{sgn}\left(p^{i} u^{\prime}\right), 0 \leq i \leq$ $G(P, \mathbf{b})$.

b) For each $u \in P \in \mathcal{P}(\mathbf{b})$, if $G(P, \mathbf{b})<\infty$, there is no $u^{\prime} \in \mathbb{C}$ such that $\operatorname{sgn}\left(T_{i}(u, \mathbf{b})\right)=\operatorname{sgn}\left(T_{i}\left(u^{\prime}, \mathbf{b}\right)\right)=$ $\operatorname{sgn}\left(p^{i} u^{\prime}\right), 0 \leq i \leq G(P, \mathbf{b})+1$.

Proof:

a) It follows from the construction of partition $\mathcal{P}(\mathbf{b})$.

b) Because for any $u^{\prime} \in P$ it is $P \subset \cup_{i=0}^{G(P, \mathbf{b})+1} F Z^{i}\left(u^{\prime}, \mathbf{b}\right)$, and for any $u^{\prime} \notin P$ there is a $k, 0 \leq k \leq G(P, \mathbf{b})+1$, such that $\operatorname{sgn}\left(T_{k}(u, \mathbf{b})\right) \neq \operatorname{sgn}\left(\left(T_{k}\left(u^{\prime}, \mathbf{b}\right)\right)\right.$.

Figs. 3 and 4 do not show any example of partition $\mathcal{P}(\mathbf{b})$ but can provide some intuition as to what is happening in these partitions. Both Figures show the lines $l_{i}((u, \mathbf{b}))$ generated by an initial condition $u=1$. In the first case, the point $u$ is clearly engulfed by the lines, and therefore $G(P, \mathbf{b})<\infty$, where $P$ is the set, $P \in \mathcal{P}(\mathbf{b})$, such that $u \in P$. In the second case, however, this is clearly not happening. In fact, it can be predicted that $1 \in Q=\{u \in \mathbb{C}:|\operatorname{Arg}(u)|<(\pi / 14)\} \in \mathcal{P}(\mathbf{b})$, and $G(Q, \mathbf{b})=\infty$. It is interesting to note that in the configuration of Fig. 4, if we change the point $u$ to many other positions, it won't be trapped by its corresponding lines $l_{i}((u, \mathbf{b}))$, because these lines rotate following $u$. In the case of lossless resonators, this will lead us later to say that this oscillator is 'tuned' to this specific frequency, whereas a similar condition, depending this time on the initial condition $u \in \mathbb{C}$, may be defined for PDOs with a leaky resonator.

Finally, the symbolic dynamics of the PDO can be easily extracted from the $3^{L}$ partitions $\mathcal{P}(\mathbf{b})$, although not in a closed 


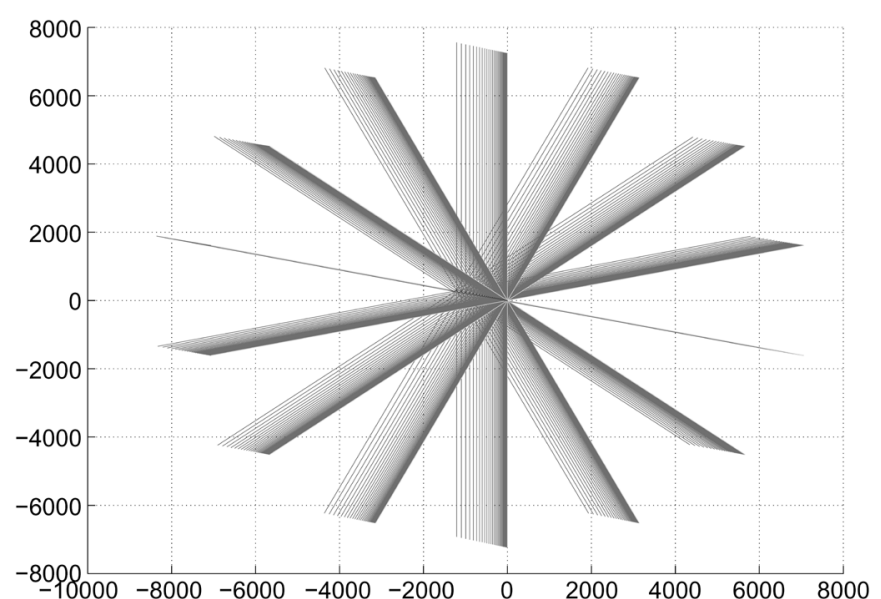

Fig. 4. Set of 250 lines $l_{0}(1,(0,0)) \cdots l_{249}(1,(0,0))$, generated by $u=1$ for an oscillator with $f=3 / 7, L=2, \delta=-1, \rho=0.005$. It is clear that point 1 is not trapped by these lines and therefore if $1 \in Q \in \mathcal{P}(\mathbf{b})$ then $G(Q, \mathbf{b})=\infty$.

form. Once an initial condition is specified for bitstream of the oscillator, $\mathbf{b} \in \mathcal{B}^{L}$, depending on the initial condition of the resonator $(u)$, the output of the oscillator will be $\operatorname{sgn}\left(p^{i} u\right), 0 \leq i \leq$ $G(P, \mathbf{b}), u \in P \in \mathcal{P}(\mathbf{b})$. Now, if $\left(u_{1}, \mathbf{b}_{1}\right)=\tau^{G(P, \mathbf{b})}(u, \mathbf{b})$, the procedure can be iterated producing a sequence of times $n_{i}$ and complex numbers, $u_{i}$, and vectors $\mathbf{b}_{i} \in \mathcal{B}^{L}$ which completely specify the bitstream at the output of the oscillator in terms of pieces of $S^{3}$ sequences.

The extensive computation of plots like those in Figs. 3 and 4 has proven to be an excellent tool to predict the results of the next Section.

\section{PRoperties of PDO SYSTEMS}

The main objective of this section is to obtain sufficient or necessary conditions under which the oscillator provides a perfect $S^{3}$ sequence for all clock cycles. First, some general properties of PDO systems are presented and then specific cases are analyzed. It must be assumed that the oscillator and the working frequency are specified through the value of $p, L$ and $\delta$.

In order to simplify the problem, we will assume throughout this section that the initial bitstream $\mathbf{b} \in \mathcal{B}^{L}$ has the following property.

Definition 7: A $b \in \mathcal{B}^{L}$ is said to be consistent with $u \in \mathbb{C}$ if $\mathbf{b}=\left(\operatorname{sgn}\left(p^{-1} u\right), \cdots, \operatorname{sgn}\left(p^{-L} u\right)\right)$.

If the initial bitstream of an oscillator is consistent with the initial value of the resonator, $u \in \mathcal{C}$, the situation is equivalent to having $\mathbf{b}=(0, \cdots, 0)$ with an initial condition of the resonator $p^{-L} u$. This assumption should be taken into account each time a reference is made to the partition $\mathcal{P}(\mathbf{b})$ or the function $G(P, \mathbf{b})$ or $T(u, \mathbf{b})$.

Lemma 7: For any given $z=(u, \mathbf{b}) \in \mathbb{C} \times \mathcal{B}^{L}$, with $\mathbf{b}$ consistent with $u$, for all $0 \leq n \leq G(P, \mathbf{b})$, with $u \in P \in$ $\mathcal{P}(\mathbf{b})$, it is

$$
\operatorname{sgn}\left(j \delta^{-1} u^{*} p^{-n+1+L} H_{n}((u, \mathbf{b}))\right) \geq 0 .
$$

Proof: Since $0 \leq n \leq G(P, \mathbf{b})$, the bit sequence for $n$ clock cycles will be $b_{n}^{\bar{L}}=\operatorname{sgn}\left(T_{n-L}(z)\right)=\operatorname{sgn}\left(p^{n-L} u\right)$. Then the Lemma follows because

$$
j \delta^{-1} u^{*} p^{-n+1+L} H_{n}((u, \mathbf{b}))=\sum_{i=0}^{n-1} u^{*} p^{-(i-L)} \operatorname{sgn}\left(p^{i-L} u\right)
$$

and Real $\left[u^{*} p^{-(i-L)} \operatorname{sgn}\left(u p^{i-L}\right)\right] \geq 0$, for all $i \in \mathbb{Z}$.

Lemma 8: For any given $z=(u, \mathbf{b}) \in \mathbb{C} \times \mathcal{B}^{L}$, with $\mathbf{b}$ consistent with $u$, for all $0 \leq n<n+N+1 \leq G(P, \mathbf{b})$, with $u \in P \in \mathcal{P}(\mathbf{b})$ and $f=M / N \in \mathbb{Q}$, it is

$$
H_{n+N+1}(z)-H_{n+1}(z)=p\left(H_{n+N}(z)-H_{n}(z)\right) .
$$

Proof: It is obvious since $H_{n+N+1}(z)=p H_{n+N}(z)-$ $j \delta \operatorname{sgn}\left(p^{n+N-L} u\right), H_{n+1}(z)=p H_{n}(z)-\operatorname{sgn}\left(p^{n-L} u\right)$ and $\operatorname{sgn}\left(p^{n+N-L} u\right)=\operatorname{sgn}\left(p^{n-L} u\right)$.

Lemma 9: Given an oscillator with $f=(M / N) \in \mathbb{Q}$, for any given $z=(u, \mathbf{b}) \in \mathbb{C} \times \mathcal{B}^{L}$, with $\mathbf{b}$ consistent with $u$, for all $0 \leq n<n+(l+1) N \leq G(P, \mathbf{b}), l \geq 0$, with $u \in P \in \mathcal{P}(\mathbf{b})$,it is

$$
H_{n+(l+1) N}((u, \mathbf{b}))=H_{n+l N}((u, \mathbf{b}))+p^{n+l N} H_{N}((u, \mathbf{b})) .
$$

Proof: It follows since

$$
\begin{aligned}
& H_{n+(l+1) N}(u, \mathbf{b})=-j \delta p^{n+(l+1) N-1} \\
& \times \sum_{i=0}^{n+(l+1) N-1} p^{-i} \operatorname{sgn}\left(p^{-L} p^{i} u\right)
\end{aligned}
$$

and

$$
H_{n+l N}(u, \mathbf{b})=-j \delta p^{n+l N-1} p^{N} \sum_{i=0}^{n+l N-1} p^{-i} p^{-N} \operatorname{sgn}\left(p^{-L} p^{i} u\right)
$$

and taking into account that for any complex number $w$, $\operatorname{sgn}\left(w p^{N}\right)=\operatorname{sgn}(w)$.

Proposition 1: For any given $z=(u, \mathbf{b}) \in \mathbb{C} \times \mathcal{B}^{L}$, with b consistent with $u$, for all $0<n \leq G(P, \mathbf{b})$, with $u \in P \in$ $\mathcal{P}(\mathbf{b})$, it is

$$
\begin{aligned}
H_{n}((u, \mathbf{b})) & =-j \delta \frac{p^{n-1}-(-1)^{\nu(n)} p^{-1}}{1-p^{-1}} \operatorname{sgn}\left(p^{-L} u\right) \\
& -j 2 \delta \frac{p^{n-1}}{1-p^{-1}} e^{-j 2 \pi \beta} \sum_{k=1}^{\nu(n)} \alpha^{-n_{k}} e^{j 2 \pi f\left\langle\frac{\langle 2 \beta\rangle-k}{2 f}\right\rangle}
\end{aligned}
$$

where the sequence $\left\{n_{k}=\lceil k-\langle 2 \beta\rangle / 2 f\rceil\right\}, 0<k \leq \nu(n)$, $\beta=\left\langle(1 / 4)-\left(\operatorname{Arg}\left\{p^{-L} u\right\} / 2 \pi\right)\right\rangle$, is formed by the time events on which $\operatorname{sgn}\left(p^{n_{k}-1} p^{-L} u\right)=-\operatorname{sgn}\left(p^{n_{k}} p^{-L} u\right)$, and $\nu(n)=$ $\lfloor 2 n f+\langle 2 \beta\rangle\rfloor$ is the number of such events in the bitstream formed by $\mathrm{n}$ bits.

Proof: $\mathbf{b}$ is consistent with $u$, and for all $0<n \leq$ $G(P, \mathbf{b}), \operatorname{sgn}\left(T_{n}((u, \mathbf{b}))\right)=\operatorname{sgn}\left(p^{n} u\right)$ which means that $b_{n}^{L}(z)=\operatorname{sgn}\left(p^{n-L} u\right), 0 \leq n \leq G(P, \mathbf{b})+L$. The main property of a sequence of bits is that it is completely determined by knowing the first bit and the instants of change (the instants when $b_{n}^{L}(z)=-b_{n-1}^{L}(z)$ ). In this case, it is $b_{n}^{L}(z)-b_{n-1}^{L}(z)=2$ only when $\langle\beta+(n-1) f\rangle>1 / 2$ and 
$\langle\beta+n f\rangle<1 / 2$, and $b_{n}^{L}(z)-b_{n-1}^{L}(z)=-2$ only when $\langle\beta+(n-1) f\rangle<1 / 2$ and $\langle\beta+n f\rangle>1 / 2$. For all other cases $b_{n}^{L}(z)-b_{n-1}^{L}(z)=0$. Then, the instants of change are those on which the sequence $(n f+\beta \bmod 1 / 2)$ decreases (the moments at which there is an 'overflow'). This means that the sequence $\left\{c_{n}=\left|b_{n}^{L}-b_{n-1}^{L}\right| / 2\right\}$ is a $\Sigma \Delta$ sequence generated by $2 f$ with initial integrator value $2 \beta$, as it was shown in Section II.

The instants of change $\left\{n_{k}:\left|b_{n_{k}}^{L}(z)-b_{n_{k}-1}^{L}(z)\right|=2\right\}$, $k>0$ with $0<n_{k} \leq n$ are such that if $n_{k}-n_{k-1}=d_{k}$ then $d_{k}$ is the minimum integer such that

$$
\left(n_{k-1} f+\beta\right) \bmod 1 / 2+d_{k} f \geq 1 / 2 .
$$

Therefore $d_{k}=\left\lceil 1-\left\langle n_{k-1} 2 f+2 \beta\right\rangle / 2 f\right\rceil, d_{k}+d_{k-1}=$ $\left\lceil 2-\left\langle n_{k-2} 2 f+2 \beta\right\rangle / 2 f\right\rceil$, and in general $n_{k}=d_{1}+\cdots+d_{k}=$ $\lceil k-\langle 2 \beta\rangle / 2 f\rceil$.

On the other hand, $\nu(n)$ is the number of edges in the bitstream from bit 0 to bit $n$. Therefore, $\nu(n)$ is the largest integer such that

$$
\left\lceil\frac{\nu(n)-\langle 2 \beta\rangle}{2 f}\right\rceil \leq n
$$

which means that $\nu(n)=\lfloor 2 f n+\langle 2 \beta\rangle\rfloor$.

Now, with $\nu(n)$ and the sequence $n_{k}$ is possible to characterize the bitstream as bursts of ' 1 's and ' -1 's.

$$
\begin{aligned}
H_{n}((u, \mathbf{b}))=- & j \delta p^{n-1} \operatorname{sgn}\left(p^{-L} u\right) \\
\times & \left(\frac{1-p^{-n_{1}}}{1-p^{-1}}-\frac{p^{-n_{1}}-p^{-n_{2}}}{1-p^{-1}}\right. \\
& \left.\quad+\frac{p^{-n_{2}}-p^{-n_{3}}}{1-p^{-1}} \cdots \frac{p^{-n_{\nu(n)}}-p^{-n}}{1-p^{-1}}\right) \\
= & -j \delta \frac{p^{n-1}}{1-p^{-1}} \operatorname{sgn}\left(p^{-L} u\right) \\
\times & \left(1-2 p^{-n 1}+2 p^{-n 2}-\right. \\
& \left.\cdots 2 p^{-n_{\nu(n)}}-(-1)^{\nu(n)} p^{-n}\right)
\end{aligned}
$$

and therefore

$$
\begin{aligned}
H_{n}((u, \mathbf{b}))= & -j \delta \frac{p^{n-1}-(-1)^{\nu(n)} p^{-1}}{1-p^{-1}} \operatorname{sgn}\left(p^{-L} u\right) \\
& -j 2 \delta \frac{p^{n-1}}{1-p^{-1}} \operatorname{sgn}\left(p^{-L} u\right) \sum_{k=1}^{\nu(n)}(-1)^{k} p^{-n_{k}}
\end{aligned}
$$

and from here expression (29) is obtained, using the fact that if $a \in \mathbb{R},\lceil a\rceil=a+\langle-a\rangle$.

Definition 8: Given a $u \in \mathbb{C}$ we define the sequence $h_{n}(u)$, $n>0$ as

$$
h_{n}(u)=-j \delta p^{n-1} \sum_{i=0}^{n-1} p^{-i} \operatorname{sgn}\left(p^{i-L} u\right)
$$

with $h_{0}(u)=0$.

Lemma 10: Given a $u \in \mathbb{C}$, if $\operatorname{sgn}\left(p^{n} u+h_{n}(u)\right)=$ $\operatorname{sgn}\left(p^{n} u\right)$, then $\operatorname{sgn}\left(T_{n}(u, \mathbf{b})\right)=\operatorname{sgn}\left(p^{n} u\right), n \geq 0$, being $\mathbf{b}$ consistent with $u$.

Proof: It follows since $\operatorname{sgn}\left(T_{n}(u, \mathbf{b})\right)=\operatorname{sgn}\left(p^{n} u+\right.$ $\left.h_{n}(u)\right)=\operatorname{sgn}\left(p^{n} u\right)$, and then $H_{n}((u, \mathbf{b}))=h_{n}(u)$, $n \geq 0$. Therefore, the sequence $\left\{\operatorname{sgn}\left(p^{n} u\right)\right\}$ is solution of the problem.

This last result will be used to find sufficient conditions for oscillations at the resonant frequency of the resonator in the next subsection.

\section{A. Sufficient Conditions for Oscillations at the Resonant Frequency of the Resonator}

Several cases will be considered. The first one corresponds to frequencies of the form $f=1 / 2 M$, on which (29) can be easily calculated, for all values of $n$. Next, the more general frequencies of the form $f=M / N$ are analyzed, using this time Lemma 9.

1) Case $f=1 / 2 M, \rho=0$ : In this case, it is

$$
2 \sin (\pi f) \delta^{-1} h_{n}(u)=\eta_{1}(n)+\eta_{2}(n)
$$

where

$$
\eta_{1}(n)=e^{j \frac{\pi}{2 M}}\left(e^{-j \frac{\pi n}{M}} \operatorname{sgn}\left(p^{-L} u\right)-(-1)^{\left\lfloor\frac{n}{M}+2 \beta\right\rfloor}\right) .
$$

Note that $\left.(-1)^{\lfloor(n / M)+\langle 2 \beta\rangle}\right\rfloor \operatorname{sgn}\left(p^{-L} u\right)=(-1)^{\lfloor(n / M)+2 \beta\rfloor}$. And

$$
\eta_{2}(n)=2\left\lfloor\frac{n}{M}+\langle 2 \beta\rangle\right\rfloor e^{-j 2 \pi \frac{n+\lfloor M 2 \beta\rfloor}{2 M}} e^{j \frac{\pi}{2 M}} .
$$

In this case it has also been used that $e^{-j 2 \pi(n+\lfloor M\langle 2 \beta\rangle\rfloor / 2 M)} \operatorname{sgn}\left(p^{-L} u\right)=e^{-j 2 \pi(n+\lfloor M 2 \beta\rfloor / 2 M)}$.

If $L=(M / 2)-1$, with $\mathrm{M}$ even, then $\operatorname{sgn}\left(\eta_{2}(n)\right)=$ $\operatorname{sgn}\left(p^{n} u\right)$ or $\operatorname{sgn}\left(\eta_{2}(n)\right)=0$. On the other hand, there are values of $u$ such that $\operatorname{sgn}\left(\eta_{1}(n)\right)=-\operatorname{sgn}\left(p^{n} u\right)$, for some $n$, but $\left|\eta_{1}(n)\right| \leq 2 \mid$. Therefore, we can state the following lemma.

Lemma 11: If $L=(M / 2)-1, M$ is even, $\left|\operatorname{Real}\left(p^{k} u\right)\right| \geq$ $|\delta|(1 / \sin (\pi / 2 M)) \approx|\delta| M / 2, k=0, \cdots, 2 M-1$, and $\mathbf{b}$ is consistent with $u$, then $\operatorname{sgn}\left(T_{n}((u, \mathbf{b}))\right)=\operatorname{sgn}\left(p^{n} u\right), n \geq 0$.

Proof: We will be using Lemma 10. Since $\left|\operatorname{Real}\left(\eta_{1}(n)\right)\right|<$ 2 and

$$
\operatorname{Real}\left(T_{n}((u, \mathbf{b}))\right)=\operatorname{Real}\left(p^{n} u+\delta \frac{\eta_{1}(n)+\eta_{2}(n)}{2 \sin (\pi / 2 M)}\right)
$$

then $\left|\operatorname{Real}\left(p^{n} u\right)\right| \geq \delta\left(\eta_{1}(n) / 2 \sin (\pi / 2 M)\right)$ and, due to the fact that $\operatorname{sgn}\left(\eta_{2}(n)\right)=\operatorname{sgn}\left(p^{n} u\right)$, then $\operatorname{sgn}\left(T_{n}((u, \mathbf{b}))\right)=$ $\operatorname{sgn}\left(p^{n} u\right)$.

Fig. 5 shows the simulation of an oscillator with $L=2$, $\rho=0, f=1 / 12, \delta=1$, with initial condition $u=20 e^{\pi / 5}$. As it can be observed the oscillator follows a perfect $S^{3}$ sequence.

2) Case $f=M / N, \rho=0$ : In this case, we will only find $h_{N}(u)$, in order to use Lemma 9. Then, because $\nu(N)=2 M$ and using the fact that for $N$ even

$$
\begin{aligned}
& \left\{\left\langle\frac{\langle 2 \beta\rangle N / 2-k N / 2}{M}\right\rangle: k=1, \cdots, M\right\} \\
& \quad=\left\{\left\langle\frac{\langle 2 \beta\rangle N / 2-k N / 2}{M}\right\rangle: k=M+1, \cdots, 2 M\right\} \\
& \quad=\left\{\frac{k+\langle\beta N\rangle}{M}: k=0, \cdots, M-1\right\}
\end{aligned}
$$




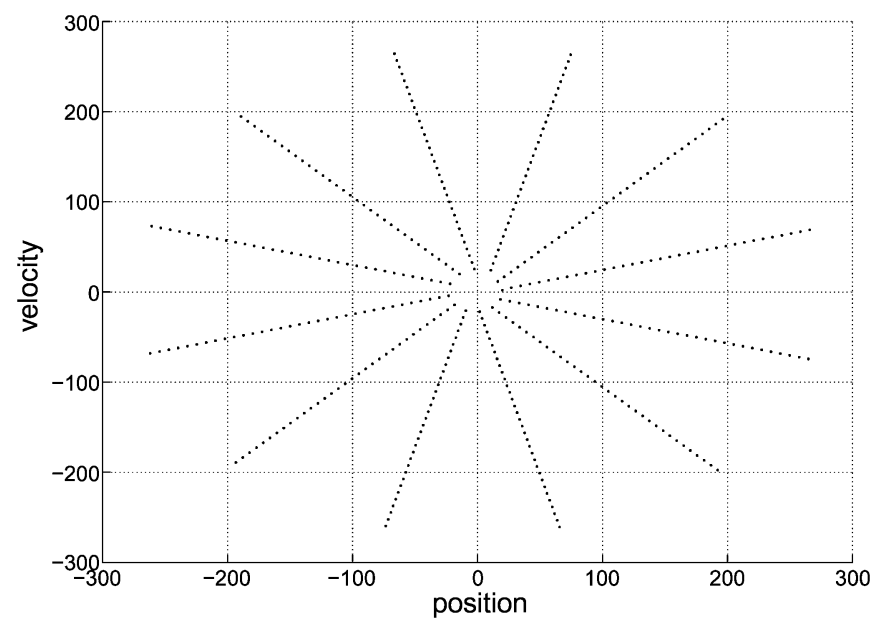

Fig. 5. Simulation of an oscillator with $L=2, \rho=0, f=1 / 12, \delta=1$, with initial condition $u=20 e^{j \pi / 5}$. Each point corresponds to $T_{n}((u, \mathbf{b}))$, with $\mathbf{b}$ consistent with $u$.

and for $N$ odd

$$
\begin{aligned}
\left\{\left\langle\frac{\langle 2 \beta\rangle N-k N}{2 M}\right\rangle: k=1, \cdots, 2 M\right\} \\
=\left\{\frac{k+\langle 2 \beta N\rangle}{2 M}: k=0, \cdots, 2 M-1\right\}
\end{aligned}
$$

then for $N$ even

$$
h_{N}(u)=2 \delta e^{j 2 \pi \frac{M}{N}} e^{-j \frac{\pi}{N}} \frac{e^{-j 2 \pi \frac{\lfloor\beta N \downarrow}{N}}}{\sin \left(\frac{\pi}{N}\right)}
$$

and for $N$ odd

$$
h_{N}(u)=\delta e^{j 2 \pi \frac{M}{N}} e^{-j \frac{\pi}{2 N}} \frac{e^{-j 2 \pi \frac{\lfloor 2 \beta N\rfloor}{2 N}}}{\sin \left(\frac{\pi}{2 N}\right)} .
$$

Now, the necessary condition to have $\operatorname{sgn}\left(T_{n}((u, \mathbf{b}))\right)=$ $\operatorname{sgn}\left(p^{n} u\right)$ is that $\operatorname{sgn}\left(p^{n} h_{N}(u)\right)=\operatorname{sgn}\left(p^{n} u\right)$. Therefore, for $N$ even it must be

$$
\frac{\left\lfloor\left(\frac{1}{4}-\frac{\operatorname{Arg}(u)}{2 \pi}\right) N\right\rfloor}{N}=\frac{\left\lfloor\left(\frac{1}{4}-\frac{\operatorname{Arg}\left(h_{N}(u)\right)}{2 \pi}\right) N\right\rfloor}{N}
$$

and for $N$ odd

$$
\frac{\left\lfloor\left(\frac{1}{4}-\frac{\operatorname{Arg}(u)}{2 \pi}\right) 2 N\right\rfloor}{2 N}=\frac{\left\lfloor\left(\frac{1}{4}-\frac{\operatorname{Arg}\left(h_{N}(u)\right)}{2 \pi}\right) 2 N\right\rfloor}{2 N} .
$$

The distinction between $N$ even or odd, comes from the fact that for $N$ odd, there are two possible bitstreams depending on the initial $u$, one in which there is one ' 1 ' more than '- -1 's and another on which the contrary happens. In order to distinguish between these two situations it is necessary to divide the phase space not in $N$ slides, but in $2 N$.

Then, it is necessary, for $\operatorname{sign}(\delta)=1$ and the following to be true.
$-N$ even $(\lfloor(N / 4)+(1 / 2)\rfloor-(L+1) M) \bmod N=0$.

$-N$ odd $(\lfloor(N / 2)+(1 / 2)\rfloor-(L+1) 2 M) \bmod 2 N=0$.

In the case $\operatorname{sign}(\delta)=-1$, the former conditions are modified as follows.

$-N$ even: $(\lfloor(3 N / 4)+(1 / 2)\rfloor-(L+1) M) \bmod N=0$.

$-N$ odd: $(\lfloor(3 N / 2)+(1 / 2)\rfloor-(L+1) 2 M) \bmod 2 N=0$.

Definition 9: A lossless oscillator is said to be tuned to a rational frequency $f=M / N$, with g.c.d. $(M, N)=1$, if the above equations hold for this given frequency, or what is the same, if $\operatorname{sgn}\left(p^{n} h_{N}(u)\right)=\operatorname{sgn}\left(p^{n} u\right), n \geq 0$, for any $u \in \mathbb{C}$.

Due to the fact that these conditions do not depend on $u$, if the oscillator is not tuned, then, for each $u \in \mathbb{C}$, there is a $0 \leq$ $n_{1}<N$ such that $\operatorname{sgn}\left(p^{n_{1}} h_{N}(u)\right)=-\operatorname{sgn}\left(p^{n_{1}} u\right)$.

When $N$ is even there is always a solution in $L$ for either $\operatorname{sgn}(\delta)=1$ or $\operatorname{sgn}(\delta)=-1$, because g.c.d. $(M, N)=1$ and therefore for any number $h_{0} \in \mathbb{Z}$ there is a $k_{0} \in \mathbb{N}$ such that $\left(h_{0}-k_{0} M\right) \bmod N=0$, in fact there are infinitely many numbers like this. When $N$ is odd, though, there will be a solution only for a given sign of $\delta$, depending on whether $\lfloor(N / 2)+$ $(1 / 2)\rfloor$ is even (then $\delta>0$ ) or odd (then $\delta<0$ ) as follows.

a) If $\lfloor(N / 2)+(1 / 2)\rfloor$ is even then there is a solution for $\delta>0,\lfloor(3 N+1) / 2\rfloor$ is odd, and therefore there is no solution for $\delta<0$.

b) If $\lfloor(N / 2)+(1 / 2)\rfloor$ is odd then there is no solution for $\delta>0,\lfloor(3 N+1) / 2\rfloor$ is even, and therefore there is a solution for $\delta<0$.

This allows us to state the following lemma.

Lemma 12: For any given $f=M / N$ there is an oscillator tuned to this frequency. If $N$ is even, regardless of $\operatorname{sign}(\delta)$ and for $N$ odd for a specific either $\delta>0$ or $\delta<0$.

Lemma 13: Any lossless oscillator is tuned to an infinite number of rational frequencies.

Proof: Given an oscillator with $\delta$ and $L$ the following are true.

a) for $\delta>0$, the oscillator is tuned to frequencies of the form $f=k /(4 k(L+1)-2)$ or $k /(4 k(L+1)-1), k>0$ and also to $f=1 / 4(L+1)$.

b) for $\delta<0$, the oscillator is tuned to $f=3 / 4(L+1)$.

c) For any $L+1$ odd, there is a solution for any $N$ (even) such that g.c.d. $(L+1, N)=1$, regardless of the sign of $\delta$.

d) For any $N$ odd, if $\delta>0$, there is a solution if g.c.d.( $L+$ $1, N)=1$ and $(N+1) / 2$ even. If $\delta<0$ there is a solution for any $N$ odd with g.c.d. $(L+1, N)=1$ and $(N+1) / 2$ odd.

Theorem 2: If a lossless oscillator is tuned to $f=M / N \in \mathbb{Q}$, $u \in \mathbb{C}$ is such that $\left|\operatorname{Real}\left(p^{n} u\right)\right|>n|\delta|, 0 \leq n<N$ and $\mathbf{b}$ is consistent with $u$, then $\operatorname{sgn}\left(T_{n}((u, \mathbf{b}))\right)=\operatorname{sgn}\left(p^{n} u\right), n \geq 0$.

Proof: We will be using Lemma 10. For any $n \geq 0$ and because $p^{N}=1$ it is

$$
h_{n}(u)=h_{n \bmod N}(u)+\left\lfloor\frac{n}{N}\right\rfloor p^{n} h_{N}(u)
$$

and $\left|\operatorname{Real}\left(p^{n} u\right)\right|>(n \bmod N)|\delta| \geq\left|h_{n} \bmod N(u)\right|$. Due to the fact that the oscillator is tuned to $f$ then $\operatorname{sgn}\left(p^{n} h_{N}(u)\right)=$ $\operatorname{sgn}\left(p^{n} u\right)$. Therefore, $\operatorname{sgn}\left(T_{n}((u, \mathbf{b}))\right)=\operatorname{sgn}\left(p^{n} u+h_{n}(u)\right)=$ $\operatorname{sgn}\left(p^{n} u\right), n \geq 0$. 


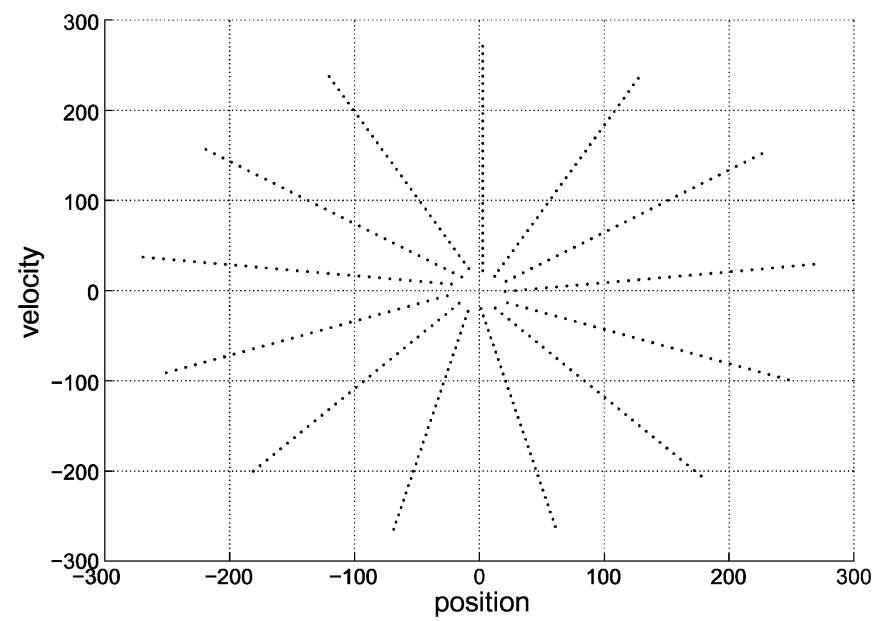

Fig. 6. Simulation of an oscillator with $L=1, \rho=0, f=5 / 13, \delta=-1$, with initial condition $u=20 e^{j \pi 13 / 45}$.

Now, as an example, we will find an oscillator tuned to $f=$ $5 / 13$. To this effect we need to solve the conditions for tuning, and a solution is $\delta<0, L=1$. Fig. 6 shows a simulation of this oscillator. As it can be seen, $S^{3}$ sequences are obtained.

Proposition 2: If the output of an oscillator, with a lossless resonator, is $\operatorname{sgn}\left(T_{n}\left(\left(u_{0}, \mathbf{b}\right)\right)\right)=\operatorname{sgn}\left(p^{n} u\right), p=\exp (-j 2 \pi f)$ for $n \geq n_{0} \geq 0$, where $f=M / N \in \mathbb{Q}$ is the resonant frequency of the resonator and $u, u_{0} \in \mathbb{C}, \mathbf{b} \in \mathcal{B}^{L}$, then the oscillator is tuned to frequency $f$.

Proof: Under these conditions, after $L$ clock cycles, $n \geq$ $n_{0}+L$, and if the value of the resonator at time $n_{0}+L$ is $u_{n_{0}+L}$, due to the constraints imposed by the PDO structure, it is $\operatorname{sgn}\left(T_{n-n_{0}-L}\left(\left(u_{n_{0}+L}, \mathbf{b}^{\prime}\right)\right)\right)=\operatorname{sgn}\left(p^{n} u\right), n \geq n_{0}+L$, with $\mathbf{b}^{\prime} \in \mathcal{B}^{L}$ consistent with $p^{n_{0}+L} u$. Then, it is $T_{n_{2}}\left(\left(u_{0}, \mathbf{b}\right)\right)-$ $T_{n_{1}}\left(\left(u_{0}, \mathbf{b}\right)\right)=h_{n_{2}}(u)-h_{n_{1}}(u)$, for any $n_{0}+L \leq n_{1}<n_{2}$, being $h_{n}(u)$ defined in Definition 8.

Let us assume that the oscillator is not tuned to $f$, then there is an $0 \leq l<N$ such that $\operatorname{sgn}\left(p^{l} h_{N}(u)\right) \neq \operatorname{sgn}\left(p^{l} u\right)$. Due to the assumptions of the proposition, for any $n_{1} \geq n_{0}+L$ such that $n_{1} \bmod N=l$ it is $\operatorname{sgn}\left(T_{n_{1}}\left(\left(u_{0}, \mathbf{b}\right)\right)=\right.$ $\operatorname{sgn}\left(p^{n_{1}} u\right)=\operatorname{sgn}\left(p^{l} u\right)$. Now, for any $K>0$ it is $T_{n_{1}+K N}\left(\left(u_{0}, \mathbf{b}\right)\right)-T_{n_{1}}\left(\left(u_{0}, \mathbf{b}\right)\right)=K p^{l} h_{N}(u)$. Using $(45)$, for a sufficiently high $K$, it will be $\operatorname{sgn}\left(T_{n_{1}+K N}\left(\left(u_{0}, \mathbf{b}\right)\right)\right)=$ $-\operatorname{sgn}\left(p^{l} u\right)$, because $\operatorname{sgn}\left(p^{l} h_{N}(u)\right)=-\operatorname{sgn}\left(p^{l} u\right)$. This means that the bitstream sequence cannot be strictly periodic of period $\mathrm{N}$ from $n_{0}$, and therefore we have a contradiction.

3) Case $f=M / N, \rho>0$ : In this case, under the conditions of Lemma 9 , for $l>0$ we have

$$
\begin{aligned}
h_{n+l N}(u)-h_{n}(u) & =p^{n} h_{N}(u) \sum_{k=0}^{l-1} \alpha^{k N} \\
& =p^{n} h_{N}(u) \frac{1-\alpha^{l N}}{1-\alpha^{N}}
\end{aligned}
$$

which means that, for $n \geq 0$

$$
h_{n}(u)=h_{n \bmod N}(u)+\frac{1-\alpha^{\left\lfloor\frac{n}{N}\right\rfloor N}}{1-\alpha^{N}} p^{n \bmod N} h_{N}(u) .
$$

Expression (47) obviously implies that now the amplitude of the oscillation remains bounded. Now, if we try the technique

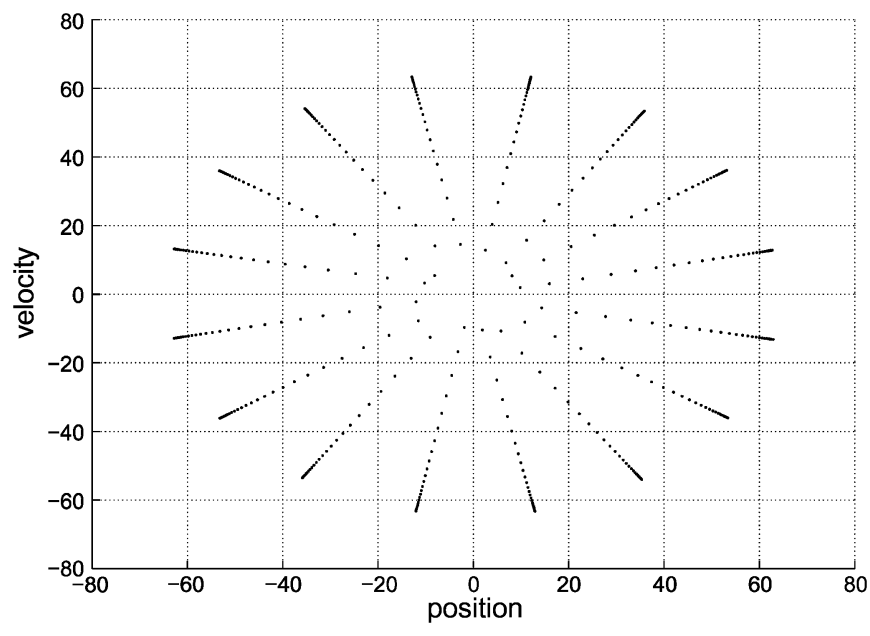

Fig. 7. Simulation of an oscillator with $L=3, \rho=0.005, f=5 / 16, \delta=1$, with initial condition $u=10 e^{j \pi / 16}$.

used in the case $\rho=0$, there is a problem with the analytical expression of $h_{N}(u)$. Recalling (29) in Proposition 1, it is not easy now to find an analytical expression of $h_{N}(u)$, due to the fact that $\alpha \neq 1$. But the following result can be stated.

Theorem 3: If for a leaky oscillator, an initial condition of the resonator $u \in \mathbb{C}$ and a frequency $f=M / N \in \mathbb{Q}$, it is for $N$ even

$$
\frac{\left\lfloor\left(\frac{1}{4}-\frac{\operatorname{Arg}(u)}{2 \pi}\right) N\right\rfloor}{N}=\frac{\left\lfloor\left(\frac{1}{4}-\frac{\operatorname{Arg}\left(h_{N}(u)\right)}{2 \pi}\right) N\right\rfloor}{N}
$$

and $N$ odd

$$
\frac{\left\lfloor\left(\frac{1}{4}-\frac{\operatorname{Arg}(u)}{2 \pi}\right) 2 N\right\rfloor}{2 N}=\frac{\left\lfloor\left(\frac{1}{4}-\frac{\operatorname{Arg}\left(h_{N}(u)\right)}{2 \pi}\right) 2 N\right\rfloor}{2 N}
$$

and $u \in \mathbb{C}, n_{0} \geq 0$ are such that the following are true.

a) $\left|\operatorname{Real}\left(p^{n} u\right)\right|>|\delta|\left(\left(1-\alpha^{n}\right) /(1-\alpha)\right), 0 \leq n<n_{0}$.

b) $\left(1-\alpha^{\lfloor n / N\rfloor N}\right) /\left(1-\alpha^{N}\right)\left|\operatorname{Real}\left(p^{n} \bmod N h_{N}(u)\right)\right|>$ $|\delta|\left(\left(1-\alpha^{N}\right) /(1-\alpha)\right), n \geq n_{0}$.

then for $b \in \mathcal{B}^{L}$ consistent with $u, \operatorname{sgn}\left(T_{n}((u, \mathbf{b}))\right)=$ $\operatorname{sgn}\left(p^{n} u\right), n \geq 0$.

Proof: We will be using Lemma 10. For $0 \leq n<n_{0}$ it is

$$
\operatorname{sgn}\left(T_{n}((u, \mathbf{b}))\right)=\operatorname{sgn}\left(p^{n} u+h_{n}(u)\right)=\operatorname{sgn}\left(p^{n} u\right)
$$

because $\left|\operatorname{Real}\left(p^{n} u\right)\right|>|\delta|\left(\left(1-\alpha^{n}\right) /(1-\alpha)\right) \geq\left|h_{n}(u)\right|$. Now, for $n \geq n_{0}$, it is

$$
\begin{aligned}
& \frac{1-\alpha^{\left\lfloor\frac{n}{N}\right\rfloor N}}{1-\alpha^{N}}\left|\operatorname{Real}\left(p^{n \bmod N} h_{N}(u)\right)\right| \\
& \geq|\delta| \frac{1-\alpha^{N}}{1-\alpha} \geq\left|\operatorname{Real}\left(h_{n \bmod N}(u)\right)\right| .
\end{aligned}
$$

Now, finally due to the fact that for this initial condition, $u$ and frequency $f$, (48), (49) hold, then $\operatorname{sgn}\left(p^{n} h_{N}(u)\right)=\operatorname{sgn}\left(p^{n} u\right)$, and therefore $\operatorname{sgn}\left(T_{n}((u, \mathbf{b}))=\operatorname{sgn}\left(p^{n} u+h_{n}(u)\right)=\right.$ $\operatorname{sgn}\left(p^{n} u\right), n \geq 0$.

An example of the behaviour predicted by this result can be found in Fig. 7. In this case, it can clearly be seen that $\operatorname{sgn}\left(T_{n+N}(u, \mathbf{b})-T_{n}(u, \mathbf{b})\right)=\operatorname{sgn}\left(p^{n} u\right), n \geq 0$. Condition b) of the result can be fulfilled for $\alpha \rightarrow 1$. 


\section{B. Detuned Lossless Oscillators With $f \in \mathbb{Q}$}

Up to this point, we have introduced a number of sufficient conditions to obtain $S^{3}$ sequences at the output of the PDO. The objective of this section is to find some characteristics of the phase-space trajectories that PDOs follow when the resonator has no damping losses and the oscillator is not tuned to a given frequency $f$. Throughout this section $f=M / N$, g.c.d. $(M, N)=1$ and the initial bitstream $\mathbf{b}$ is adapted to the initial condition of the resonator.

In order to simplify notation we will use $H_{n}$ and $b_{k}^{i}$ instead of $H_{n}((u, \mathbf{b}))$ and $b_{k}^{i}((u, \mathbf{b}))$, without making explicit reference to $(u, \mathbf{b}) \in \mathbb{C} \times \mathcal{B}^{L}$.

Lemma 14: Let us assume there exists an infinite sequence, $S=\left\{n_{i}: i \in \mathbb{N}, n_{i}<n_{i+1}\right\}$, of all time events on which $b_{n_{i}}^{1}=-b_{n_{i}+N}^{1}$. For each $n_{i} \in S$, if $n_{i} \bmod N=k$ and $n_{j}$ is the next element in $S$ such that $n_{j} \bmod N=k$, then $b_{n_{i}}^{1}=-b_{n_{j}}^{1}$.

Proof: From the hypothesis $n_{i}-n_{j}=K N$, for a given $K>0$. Since $n_{j}$ is the next element in $S$ with $b_{n_{j}+N}^{1}=$ $-b_{n_{j}}^{1}$, then $b_{n_{i}+m N}^{1}=-b_{n_{i}}^{1}, 1 \leq m \leq K$. Therefore, $b_{n_{j}}^{1}=$ $b_{n_{i}+K N}^{1}=-b_{n_{i}}^{1}$

Corollary 3: In the same conditions of Lemma 14, for all $k>0$

$$
H_{n+k N+L}-H_{n+L}=j 2 \delta p^{-1} \sum_{l=0}^{N-1} c_{l} p^{-l}
$$

where $c_{l}=0$ if the number of elements of $S$ such that $n_{i} \bmod N=l$, and $n_{i} \in\{n, n+1, \cdots, n+k N-1\}$ is even. And $c_{l}=b_{n_{l}}^{1}$ otherwise, where $n_{l}$ is the first element in $S \cap\{n, \cdots, n+k N-1\}$ such that $n_{l} \bmod N=l$.

Proof: From the definition of $H_{n}$, and taking into account that $p^{N}=1$ (lossless resonator) it is

$$
H_{n+L}=-j \delta p^{-1} \sum_{i=0}^{N-1} p^{-i} b_{n+i}^{1}
$$

and therefore, for $k>0$

$$
H_{n+k N+L}-H_{n+L}=j 2 \delta p^{-1} \sum_{n \leq n_{i} \in S<n+k N} p^{-n_{i}} b_{n_{i}}^{1}
$$

ans the result follows using Lemma 14.

Corollary 4: In the same conditions of Lemma 14, if $\left\{n_{i} \bmod N: i=k, k+1, \cdots, k+N-1\right\}=\{0, \cdots, N-1\}$, for all $k \geq 0$, then

$$
H_{n_{i+2 N}+L}=H_{n_{i}+L} \quad \forall n_{i} \in S .
$$

If the above property holds for an lossless oscillator, then it is said to possess two 'spirals'.

Proof: Following the same notation of Corollary 3

$$
H_{n_{i+2 N}+L}-H_{n_{i}+L}=\frac{2 F}{m \omega_{0}} p^{n_{i+2 N}-n_{i}} \sum_{l=0}^{N-1} c_{l} p^{l} .
$$

If $\left\{n_{i} \bmod N: i=k, k+1, \cdots, k+N-1\right\}=\{0, \cdots, N-1\}$, for all $k \geq 0$, then for each $l \in\{0, \cdots, N-1\}$ the number of

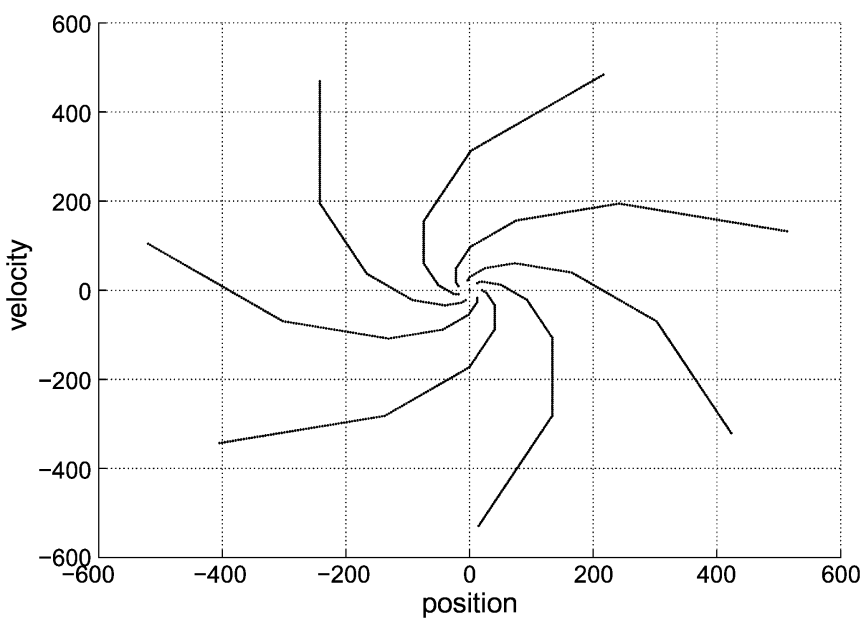

Fig. 8. Simulation of a two-spiral oscillator with $L=1, \rho=0, f=4 / 7$, $\delta=1$, with initial condition $u=20 e^{j \pi 13 / 45}$.

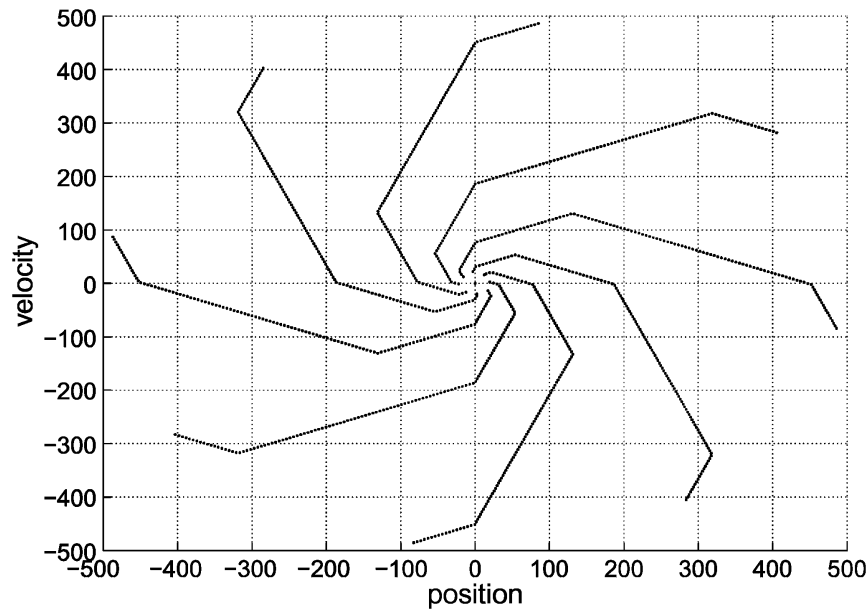

Fig. 9. Simulation of a two-spiral oscillator with $L=2, \rho=0, f=3 / 8$, $\delta=1$, with initial condition $u=20 e^{j \pi 13 / 45}$.

elements of $S$ such that $n_{i} \in\left\{n_{i}-L, \cdots, n_{i+2 N}-L\right\}$ and $n_{i} \bmod N=l$, is even, and therefore $c_{l}=0$.

Fig. 8 shows an oscillator with two spirals. It is interesting to note that for frequencies with $N$ even, though, there is an 'optical' effect on the trajectories so that it seems to be

$$
H_{n_{i+N}+L}=H_{n_{i}+L} \quad \forall n_{i} \in S
$$

which is not true. This effect can clearly be seen in Fig. 9. What it is really happening, is that for each $n_{i} \in S$ either $n_{i}-n_{i-1} \leq$ $N$ or $n_{i+1}-n_{i} \leq N$. Therefore, each time we observe a change in the vectors $H_{n}$ it is not due to a single event $n_{i}$, but to two of them.

Finally, it is also interesting to note that in order to 'observe' the same two-spiral pattern in the evolution of the oscillator it is not necessary that the conditions of Corollary 4 are strictly fulfilled. Sometimes, translocations occur and for example a number is repeated in $\left\{n_{i} \bmod N: i=k, k+1, \cdots, k+N-1\right\}$, for a given $k$, then in the next $N$ clock cycles this number will not appear and will appear twice the missing one. 


$$
a_{l}= \begin{cases}2 \frac{\langle(\lambda-1 / 2) N\rangle-\langle\lambda N\rangle}{N}, & \text { if } I(l)=0 \\ \frac{2}{N} \frac{e^{j 2 \pi I(l)}\left(\lambda-\frac{\langle\lambda N\rangle}{N}\right)}{1-e^{-j 2 \pi I(l) / N}}\left[1-(-1)^{I(l)} e^{\left.-j 2 \pi \frac{I(l)}{N}\left(\left\langle\frac{1}{2} N\right\rangle-\xi\left(\lambda N, \frac{N}{2}\right)\right)\right],},\right. & \text { if } I(l) \neq 0\end{cases}
$$

\section{CONCLUSION}

It has been proved that a bijection exists between the sequences of first-order $\Sigma \Delta$ modulators and the sign of sampled sinusoid sequences, when no distinction is made between $s$ and $-s$, with $s$ being an $S^{3}$ sequence. This has provided a perfect tool for obtaining the oscillation frequency of PDOs directly from their bitstreams. It has also been proved that under some conditions, PDOs bitstreams are $S^{3}$ sequences at the resonant frequency of the resonator, for both lossless and leaky resonators. It has also been explained the typical "second spiral" trajectories of PDOs actuating lossless resonators.

Finally, these results seem to indicate that there is far more similarity between PDOs and first-order $\Sigma \Delta$ modulators, than with bandpass $\Sigma \Delta$ modulators. This might provide a clue as to why the oscillator response with leaky resonators looks like a distorted version of the Devil's staircase fractal, which is the response of first-order $\Sigma \Delta$ modulators with leaky integration. These results also settle the question as to why the spectra at the output of PDOs have not the usual noise-shaping characteristic of $\Sigma \Delta$ modulators.

\section{APPENDIX \\ SPECTRA OF $S^{3}$ SEQUENCES}

In this section we will consider sequences of the form

$$
s_{n}=\operatorname{sign}(\sin (2 \pi f n+2 \pi \lambda), \quad n \geq 0, f, \lambda \in[0,1)
$$

which can also be expressed as

$$
s_{n}=2(\langle n f-1 / 2+\lambda\rangle-\langle n f+\lambda\rangle) .
$$

\section{A. Case $f \in \mathbb{Q}$}

Let us assume that $f=M / N$ with $g . c . d .(M, N)=1$. Then $s_{n}$ is of period $N$.

The Fourier coefficients are usually calculated as

$$
a_{l}=\frac{1}{N} \sum_{n=0}^{N-1} s_{n} e^{-j 2 \pi l n / N}, \quad l=0 \ldots N-1 .
$$

On the other hand ([13])

$$
A=\left\{\left\langle n \frac{M}{N}\right\rangle: n=0 \ldots N-1\right\}=\left\{\frac{n}{N}: n=0 \ldots N-1\right\}
$$

which means that a bijection, $I(l)$, exists in $\{0 \ldots N-1\}$ such that

$$
I(l)=k \quad \text { and } \quad\langle k f\rangle=l / N, \quad k, l \in\{0 \ldots N-1\} .
$$

It is immediate that $I(M)=1$. Therefore

$$
\begin{aligned}
a_{l}= & 1 \frac{1}{N} \sum_{n=0}^{N-1} s_{n} e^{-j 2 \pi\langle I(l) f\rangle n}=\frac{1}{N} \sum_{n=0}^{N-1} s_{n} e^{-j 2 \pi I(l)\langle n f\rangle} \\
= & \frac{2}{N} \sum_{n=0}^{N-1}\left(\left\langle n f-\frac{1}{2}+\lambda\right\rangle-\langle n f+\lambda\rangle\right) \\
& \times e^{-j 2 \pi I(l)\langle n f\rangle} .
\end{aligned}
$$

Taking into account that for $y \in \mathbb{R}$

$$
\begin{aligned}
& \{\langle n f+y\rangle: n=0 \ldots N-1\} \\
& \quad=\left\{\langle n f\rangle+\frac{\langle y N\rangle}{N}: n=0 \ldots N-1\right\} \\
& \quad=\left\{\frac{n}{N}+\frac{\langle y N\rangle}{N}: n=0 \ldots N-1\right\}
\end{aligned}
$$

then

$$
\begin{aligned}
a_{l}=\frac{2 e^{j 2 \pi I(l) \lambda}}{N} \sum_{n=0}^{N-1}\{ & (-1)^{I(l)}\left\langle n f-\frac{1}{2}+\lambda\right\rangle \\
& \times e^{-j 2 \pi I(l)\left\langle n f-\frac{1}{2}+\lambda\right\rangle} \\
& \left.-\langle n f+\lambda\rangle e^{-j 2 \pi I(l)\langle n f+\lambda\rangle}\right\} \\
=\frac{2 e^{j 2 \pi I(l) \lambda}}{N} & \left\{(-1)^{I(l)} \sum_{n=0}^{N-1}\left(\frac{n}{N}+\frac{\left\langle\left(-\frac{1}{2}+\lambda\right) N\right\rangle}{N}\right)\right. \\
& \times e^{-j 2 \pi I(l)\left(\frac{n}{N}+\frac{\left\langle\left(-\frac{1}{2}+\lambda\right) N\right\rangle}{N}\right)} \\
& \left.-\sum_{n=0}^{N-1}\left(\frac{n}{N}+\frac{\langle\lambda N\rangle}{N}\right) e^{-j 2 \pi I(l)\left(\frac{n}{N}+\frac{\langle\lambda N\rangle}{N}\right)}\right\} .
\end{aligned}
$$

Therefore, the equation (66), shown at the top of this page is true, where for $a, b \in \mathbb{R}, \xi(a, b)=1$ if $\langle a\rangle+\langle b\rangle \geq 1$ and $\xi(a, b)=0$, otherwise.

Two different cases can be distinguished: $N$ even, $N$ odd.

\section{B. Case N Even}

In this case, $\langle(1 / 2) N\rangle=0$.

$$
a_{l}= \begin{cases}0, & \text { if } I(l)=0, \text { or even } \\ \frac{4}{N} \frac{e^{j 2 \pi I(l)}\left(\lambda-\frac{\lambda \lambda N\rangle}{N}\right)}{1-e^{-j 2 \pi I(l) / N},}, & \text { if } I(l) \text { odd. }\end{cases}
$$

\section{Case N Odd}

In this case, $\langle \pm(1 / 2) N\rangle=1 / 2$. 


$$
\begin{aligned}
& \text { If }\langle\lambda N\rangle \in[0,1 / 2) \\
& a_{l}= \begin{cases}\frac{1+2\langle\lambda N\rangle}{N}, & \text { if } I(l)=0 \\
\frac{2}{N} \frac{e^{j 2 \pi I(l)}\left(\lambda-\frac{\langle\lambda N\rangle}{N}\right)}{1-e^{-j 2 \pi I(l) / N}} & \\
\quad \times\left[1-(-1)^{I(l)} e^{-j \pi \frac{I(l)}{N}}\right], & \text { if } I(l) \neq 0\end{cases}
\end{aligned}
$$

and if $\langle\lambda N\rangle \in[1 / 2,1)$

$a_{l}= \begin{cases}\frac{1+2\langle\lambda N\rangle}{N}, & \text { if } I(l)=0 \\ \frac{2}{N} \frac{e^{j 2 \pi I(l)}\left(\lambda-\frac{\langle\lambda N\rangle}{N}\right)}{1-e^{-j 2 \pi I(l) / N}}\left[1-(-1)^{I(l)} e^{j \pi \frac{I(l)}{N}}\right], & \text { if } I(l) \neq 0 .\end{cases}$

The phase of component of the fast Fourier transform (FFT) at frequency $\langle f\rangle$ is quantized. This result is in concordance with what in [2] was called the phase uncertainty of the signal, $u(f)$ (the length of the maximum interval in which $\lambda$ can change without chaging the sign of its samples). For $N$ even $u(f)=$ $1 / N$ and for $N$ odd is $u(f)=1 / 2 N$.

With the above computed Fourier coefficients it is possible to construct the bitstream in the usual way

$$
s_{n}=\sum_{n=0}^{N-1} a_{l} e^{-j 2 \pi \ln / N} .
$$

\section{Case $f \in \mathbb{R}-Q$}

In this case, and following the approach taken in [13], we will use a Bohr-Fourier representation series to describe the aperiodic bitstream sequence. It is known that, for $y \in \mathbb{R}$

$$
\langle n f+y\rangle=\sum_{k=-\infty}^{\infty} d_{k} e^{j 2 \pi n\langle k f\rangle}
$$

where

$$
\begin{aligned}
d_{k} & =\lim _{n \rightarrow \infty} \frac{e^{j 2 \pi k y}}{n} \sum_{l=0}^{n-1}\langle l f+y\rangle e^{-j 2 \pi k\langle l f+y\rangle} \\
& =e^{j 2 \pi k y} \int_{0}^{1} f e^{-j 2 \pi k f} d f
\end{aligned}
$$

due to the fact that the trajectory of $\langle n f+y\rangle$ is dense in [0,1], for $f$ irrational. Then

$$
d_{k}= \begin{cases}\frac{1}{2}, & \text { if } k=0 \\ \frac{j}{2 \pi k} e^{j 2 \pi k y}, & \text { if } k \neq 0\end{cases}
$$

The coeficients $a_{k}$ of the bitstream $s_{n}=2(\langle n f-(1 / 2)+$ $\lambda\rangle-\langle n f+\lambda\rangle)$ are then

$$
a_{k}= \begin{cases}0, & \text { if } k=0, \text { or even } \\ \frac{-2 j}{\pi k} e^{j 2 \pi k \lambda}, & \text { if } k \text { odd, frequency }\langle k f\rangle .\end{cases}
$$

In all cases, the maximum of the above calculated spectra is located at frequency $f$, regardless of whether $f$ is irrational or rational. This provides an explanation as to why in the first works on PDOs [1], [2], the method used to infer the oscillation frequency of the oscillator, by finding the frequency at which the FFT of the bitstream presented its maximum, worked properly.

\section{REFERENCES}

[1] M. Dominguez, J. Pons-Nin, J. Ricart, A. Bermejo, and E. FiguerasCosta, "A novel $\Sigma \Delta$ Pulsed Digital Oscillator (PDO) for MEMS," IEEE Sensors J., vol. 5, no. 6, pp. 1379-1388, 2005.

[2] M. Dominguez, J. Pons-Nin, J. Ricart, A. Bermejo, E. F. Costa, and M. Morata, "Analysis of the $\Sigma \Delta$ pulsed digital oscillator for MEMS," IEEE Trans. Circuits Syst. I, Reg. Papers, vol. 52, no. 11, pp. 2286-2297, Nov. 2005.

[3] M. Dominguez, J. Pons-Nin, J. Ricart, and E. Figueras, "The MEMS pulsed digital oscillator (PDO) below the Nyquist limit," Sens. Actuat. A, Phys., to be published.

[4] M. Dominguez, J. Pons, J. Ricart, J. Juillard, and E. Colinet, "Linear analysis of the influence of FIR feedback filters on the response of the pulsed digital oscillator," Anal. Integr. Circuits Syst., to be published.

[5] J. Zhang, J. Zhou, and A. Mason, "Highly adaptive transducer interface circuit for multiparameter microsystems," IEEE Trans. Circuits Syst. I, Fundam. Theory Appl., vol. 54, no. 1, pp. 167-178, Jan. 2007.

[6] J. Wu and L. R. Carley, "Electromechanical S-D modulation with high-Q micromechanical accelerometers and pulse density modulated force feedback," IEEE Trans. Circuits Syst. I, Reg. Papers, vol. 53, no. 2, pp. 274-287, Feb. 2007

[7] T. Endo and L. O. Chua, "Symbolic dynamics of piecewise-linear maps," IEEE Trans. Circuits Syst. I, Fundam. Theory Appl., vol. 19, no. 7, pp. 1485-1501, 1999.

[8] X.-C. Fu and P. Ashwin, "Symbolic analysis for some planar piecewise-linear maps," Discr. Contin. Dynam. Syst., vol. 9, no. 6, pp. 1533-1548, 2003.

[9] A. Goetz, "Sofic subshifts and piecewise-isometric systems," Ergod. Theory Dynam. Syst., vol. 19, pp. 1485-1501, 1999.

[10] C. Kahlert and L. O. Chua, "The complete canonical piecewise-linear representation. I. The geometry of the domain space," IEEE Trans. Circuits Syst. I, Fundam. Theory Appl., vol. 39, no. 2, pp. 222-236, Feb. 1992.

[11] R. Lum and L. O. Chua, "Generic properties of continuous piecewiselinear vector fields in $R^{2}$," IEEE Trans. Circuits Syst., vol. 38, no. 12, pp. 1043-1066, Dec. 1991

[12] R. M. Gray, "Oversampled sigma-delta modulation," IEEE Trans. Commun., vol. COM-35, no. 4, pp. 481-489, Apr. 1987.

[13] R. M. Gray, "Spectral analysis of quantization noise in a single-loop sigma-delta modulator with dc input," IEEE Trans. Commun., vol. 37, no. 5, pp. 588-599, May 1989.

[14] V. Lakshmikantham, D. D. Bainov, and P. S. Simeonov, Theory of Impulsive Differential Equations. Singapore: Word Scientific, 1989.

[15] N. T. Thao, "Vector quantization analysis of $\Sigma \Delta$ modulation," IEEE Trans. Signal Proc., vol. 44, no. 4, pp. 808-817, Apr. 1996.

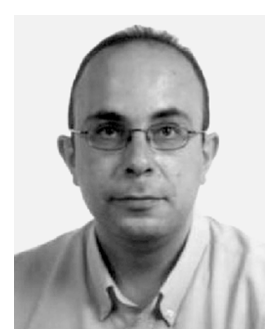

Manuel Domínguez (M’98), received the M.Sc. and $\mathrm{Ph} . \mathrm{D}$. degrees in electronic engineering from the Universitat Politècnica de Catalunya (UPC), Barcelona, Spain, in 1994 and 1997, respectively, and the degree in mathematics (with honors) from the Universidad Nacional de Educación a Distancia (UNED), Madrid, Spain in 2005.

He works in the Department of Electronic Engineering, UPC, since 1994, where he is now an Associate Professor. He was Visiting Scholar at the Courant Institute of Mathematical Sciences from September 2006 to August 2007. His research areas include the design and development of microelectromechanical systems (MEMS) sensors and actuators, sensors for space applications, sigma-delta modulation applied to MEMS, oscillators, and nonlinear circuits in general. 


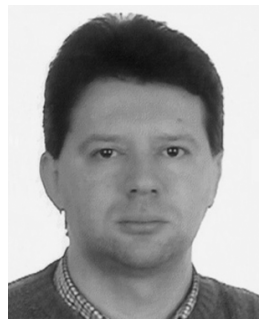

Joan Pons-Nin (M'94) received the Ingeniero Superior de Telecomunicación and the Ph.D. degrees from the Universidad Politécnica de Catalunya (UPC), Barcelona, Spain, in 1989 and 1995, respectively.

$\mathrm{He}$ is an Associate Professor in the Departamento de Ingeniería Electrónica de la Escuela Técnica Superior de Ingeniería de Telecomunicación , UPC. $\mathrm{He}$ is currently working on oscillators for microelectromechanical systems resonators. He worked also on emitter characterization and optimization for advanced bipolar devices and polysilicon contacted silicon solar cells. Semiconductor devices simulation and digital control circuitry are also research topics of his interest.

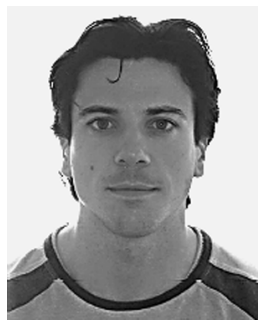

Jordi Ricart was born in Madrid, Spain, in 1975. He received the M.Sc. degree in ingeniería electrónica from the Universitat Politècnica de Catalunya (UPC), Barcelona, Spain, in 2004, where he is currently working toward the Ph.D. degree.

$\mathrm{He}$ joined the Semiconductor Devices Group, Electronic Engineering Department, UPC, as both a Research Assistant and Ph.D. degree student. He is actively working in sensors for space applications and nonlinear circuits for microelectromechanical applications, such as oscillators. 